TOKYO J. MATH.

VoL. 13, No. 1, 1990

\title{
Homogeneous Harmonic Maps into Complex Projective Spaces
}

\author{
Yoshihiro OHNITA
}

Tokyo Metropolitan University

Dedicated to Professor Tadashi Nagano on his sixtieth birthday

\section{Introduction.}

Let $M=G / K$ be a compact homogeneous space of a compact Lie group $G$ with a $G$-invariant Riemannian metric $g$ and $N$ be a Riemannian manifold. A homogeneous harmonic map from $M$ to $N$ means a $\rho$ equivariant harmonic map from $M$ to $N$ relative to a homomorphism $\rho$ of $G$ to the isometry group of $N$. The existence and construction of harmonic maps are interesting and important problems in various situations. Homogeneous harmonic maps make a simple and nice class of harmonic maps. Concerning the existence of homogeneous harmonic maps, in general it is known by the idea of W.-Y. Hsiang that given a nonconstant $\rho$-equivariant map $\varphi$ from a compact homogeneous Riemannian manifold $M$ to a compact Riemannian manifold $N$, then $\phi$ can be deformed to a nonconstant $\rho$-equivariant harmonic map $\varphi_{1}$ through a smooth homotopy of $\rho$-equivariant maps $\varphi_{t}(t \in[0,1])$ with $\varphi_{0}=\varnothing$ (cf. [Gu 5]). Naturally we are interested in getting more explicit descriptions and detailed properties of homogeneous harmonic maps for specific homogeneous Riemannian manifolds $M$ and $N$. In his nice paper [Gu 1], Guest pointed out many interesting connections of the research for homogeneous harmonic maps with problems of differential geometry and mathematical physics. Moreover he gave algebraic descriptions of the harmonic map equation for general homogeneous maps, and discussed the harmonicity of homogeneous maps into complex projective spaces and a construction of homogeneous harmonic maps from flag manifolds into complex Grassmann manifolds by the method of osculating flags and twistor spaces (cf. [E-W]). In this paper we discuss homogeneous harmonic maps and minimal immersions into complex projective spaces in detail, by using 
the relation between complex line bundles and smooth maps into complex projective spaces.

In Section 1 we shall recall the correspondence of a smooth map from a manifold into a complex projective space with a system of smooth sections for a complex line bundle over the manifold. We point out a relationship between the homotopy-theoretic properties of the smooth maps and the equivalence property of complex line bundles. Furthermore we shall describe the harmonic map equation for a smooth map into a complex projective space in terms of the corresponding system of smooth sections for a complex line bundle. In Section 2 we shall consider homogeneous complex line bundles over compact homogeneous spaces. Using the spectral decomposition of the space of all smooth sections of a homogeneous line bundle and results in Section 1, we give a description of all homogeneous maps into complex projective spaces. Furthermore we shall show a result on the multiplicity in the spectral decomposition over compact symmetric spaces, which plays an essential role in Section 5. In Section 3 we shall give a description of homogeneous harmonic maps from certain compact homogeneous Riemannian manifolds into complex projective spaces in terms of Sections 1 and 2. In Section 4 we shall apply results of Section 3 to the case when the domain manifold is a compact homogeneous Kähler manifold and study some properties of homogeneous harmonic maps from such complex manifolds into complex projective spaces. In Section 5 we shall determine the spectral decompositions for all homogeneous complex line bundles over every irreducible Hermitian symmetric space $M=G / K$ of compact type. We shall give a complete list of irreducible representations of the compact Lie group $G$ appearing in the space of smooth sections of each complex line bundle. In Section 6 we shall classify homogeneous harmonic maps and equivariant minimal isometric immersions between complex projective spaces. By this result we find a nice series of minimal isometric immersions of complex projective spaces into complex projective spaces, which contains neither holomorphic, antiholomorphic nor totally real immersions. It is a generalization of homogeneous minimal 2-spheres in complex projective spaces given by [B-O], [B-J-R-W], [Gu 1] to higher dimension. In Section 7 we shall give a remark on homogeneous minimal 2-spheres in quaternionic projective spaces.

In the famous paper [Ta], Takahashi showed a connection of the Laplacian acting on smooth functions with minimal immersions into spheres and a construction of minimal immersions of compact homogeneous Riemannian manifolds with the irreducible isotropy representation by eigenfunctions of the Laplacian. This work can be regarded also as 
a natural extension of Takahashi's results to eigensections of the Laplacian in complex line bundles and harmonic maps, minimal immersions into complex projective spaces.

We hope to find more properties and more explicit descriptions of harmonic maps and minimal immersions into complex projective spaces than those obtained here.

$\S 1$. Complex line bundles and harmonic maps into a complex projective space.

Let $C P^{n}(c)$ be an $n$-dimensional complex projective space with the Fubini-Study metric $g_{c}$ of constant holomorphic sectional curvature $c>0$ and $\boldsymbol{R} P^{n}(c)$ be an $n$-dimensional real projective space with the standard metric of constant sectional curvature $c>0 . R P^{n}(c / 4)$ is imbedded into $\boldsymbol{C P}{ }^{n}(c)$ in the natural manner as a totally real totally geodesic submanifold. Let $S^{n}(c)$ be an $n$-dimensional sphere with the standard metric of constant sectional curvature $c>0$. Then we have a natural isometric covering $\boldsymbol{S}^{n}(\boldsymbol{c}) \rightarrow \boldsymbol{R} P^{n}(c)$.

Let $\left\{\varepsilon_{0}, \cdots, \varepsilon_{n}\right\}$ be the standard basis of $C^{n+1}$. We denote by $\langle$, the standard Hermitian inner product on $C^{n+1} ;\langle z, w\rangle=\sum_{i=0}^{n} z^{i} \bar{w}^{i}$. Let $()=,\operatorname{Re}\langle$,$\rangle denote the associated real inner product on C^{n+1}$. Let $\pi: \boldsymbol{C}^{n+1} \backslash\{0\} \rightarrow C P^{n}$ be the canonical projection. Then $\boldsymbol{C}^{n+1} \backslash\{0\}$ is a principal bundle over $C P^{n}$ with the structure group $C^{*}$, where $C^{*}$ denotes the group of non-zero complex numbers. The restriction of $\pi$ to $S^{2 n+1}(c / 4)$ is a Riemannian submersion. Let $E=\left(C^{n+1} \backslash\{0\}\right) \times{ }_{C^{*}} C$ be the universal bundle over $C P^{n}$ : the fibre $E_{x}$ over any $x \in C P^{n}$ is the complex 1-dimensional subspace of $C^{n+1}$ determined by $x$. Thus $E$ is a holomorphic subbundle of the trivial bundle $\underline{C}^{n+1}$ over $\boldsymbol{C P}^{n}$. Let $E^{\perp}$ be the subbundle of $\underline{C}^{n+1}$ whose fibre at $x$ is the orthogonal complement of $E_{x}$ in $\underline{C}^{n+1}$. $E, E^{*}$ and $E^{\perp}$ have natural Hermitian connected structures. We give $E^{*} \otimes E^{\perp}$ the tensor product Hermitian connected structure. Then there exists a natural bundle isomorphism $h: T^{(1,0)} C P^{n} \rightarrow E^{*} \otimes E^{\perp}$ preserving connections which satisfies $\langle h(Z), h(W)\rangle=(c / 2) g_{c}(Z, W)$ for $Z, W \in \boldsymbol{T}_{x}^{(1,0)} \boldsymbol{C} P^{n}$ (cf. [E-W, p. 224]).

Let $\varphi: M \rightarrow C P^{n}$ be a smooth map from a manifold $M$ to a complex projective space. A map $\varphi$ is called full if the image $\varphi(M)$ is not contained in any proper complex projective subspace of $C P^{n}$. Consider the exact sequence of pull-back vector bundles over $M$ :

$$
0 \longrightarrow \varphi^{-1}\left(E^{*} \otimes E\right) \stackrel{i}{\longrightarrow} \varphi^{-1}\left(E^{*} \otimes \underline{C}^{n+1}\right) \stackrel{j}{\longrightarrow} \varphi^{-1}\left(E^{*} \otimes E^{\perp}\right) \longrightarrow 0,
$$

where $i$ is the natural inclusion and $j$ is given by the orthogonal pro- 
jection along $E$. We call the section $\Phi=i(1) \in C^{\infty}\left(\Phi^{-1}\left(E^{*} \otimes \underline{C}^{n+1}\right)\right)$ the universal lift of $\varphi$ (cf. [E-W]). Pulling back $h: T^{(1,0)} C P^{n} \rightarrow E^{*} \otimes E^{\perp}$ by $\varphi$, we get a connection-preserving bundle isomorphism $h: \varphi^{-1}\left(T^{(1,0)} C P^{n}\right) \rightarrow$ $\Phi^{-1}\left(E^{*} \otimes E^{\perp}\right)$. Let $D$ denote the covariant differentiation of the bundle $\Phi^{-1}\left(E^{*} \otimes \underline{C}^{n+1}\right)$ relative to the pull-back connection. Then the following facts are known (cf. $[\mathrm{E}-\mathrm{W}])$ :

(i) $\langle\Phi, \Phi\rangle \equiv 1$.

(ii) For any $X \in C^{\infty}\left(T M^{c}\right), \quad D_{X} \Phi \in C^{\infty}\left(\varphi^{-1}\left(E^{*} \otimes \underline{C}^{n+1}\right)\right)$ has image in $\varphi^{-1} E^{\perp}$. Moreover one has

$$
h\left((d \varphi)^{(1,0)}(X)\right)=D_{X} \Phi
$$

for any $X \in T_{x} M^{c}$. Here $(d \varphi)^{(1,0)}$ denotes the $(1,0)$-component of $d \varphi$ in $C P^{n}$.

We recall the relation between a map to a complex projective space and a system of sections of a complex line bundle.

Let $L=P \times_{\sigma} C$ be a complex line bundle over a (paracompact) manifold $M$ associated with a principal bundle $(P, \pi, M, K)$. Here $(\sigma, C)$ is a complex 1-dimensional representation of the structure group $K$. Then the vector space $C^{\infty}(L)$ of all smooth sections of $L$ can be identified with the vector space $C^{\infty}(P, C)_{K}$ of all $C$-valued smooth functions $\tilde{f}$ on $P$ satisfying the condition $\widetilde{f}(u k)=\sigma(k)^{-1} \widetilde{f}(u)$ for any $u \in P$ and $k \in K$, by the correspondence $A: C^{\infty}(L) \ni f \mapsto \tilde{f} \in C^{\infty}(P, C)_{K}, \tilde{f}(u)=u^{-1}(f(\pi(u)))$ for each $u \in P$.

Assume given a system $\left\{\varphi_{0}, \cdots, \varphi_{n}\right\}$ in $C^{\infty}(L)$ with no common zeros. Let $\left\{\widetilde{\varphi}_{0}, \cdots, \widetilde{\varphi}_{n}\right\}$ be the corresponding system in $C^{\infty}(P, C)_{K}$. We define a smooth map $\widetilde{\varphi}: P \rightarrow C^{n+1} \backslash\{0\}$ by $\widetilde{\varphi}=\left(\widetilde{\varphi}_{0}, \cdots, \widetilde{\varphi}_{n}\right)$. Since $\widetilde{\varphi}$ satisfies $\widetilde{\varphi}(u k)=$ $\sigma(k)^{-1} \widetilde{\varphi}(u)$ for any $u \in P$ and $k \in K$, the map $\widetilde{\varphi}: P \rightarrow C^{n+1} \backslash\{0\}$ becomes a bundle homomorphism from $(P, \pi, M, K)$ to $\left(C^{n+1} \backslash\{0\}, \pi, C P^{n}, C^{*}\right)$ with the homomorphism $\sigma^{-1}: K \rightarrow C^{*}$ of the structure groups. Therefore $\widetilde{\Phi}$ induces a smooth map $\varphi: M \rightarrow C P^{n}$ and the diagram

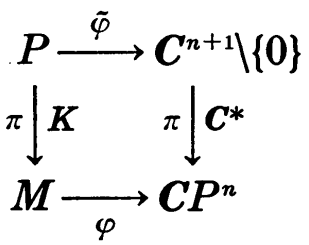

is commutative. Let $H=E^{*}$ be the hyperplane bundle over $C P^{n}$. Then we see that the pull-back bundle $\varphi^{-1} H$ is isomorphic to $L: \varphi^{-1} H \equiv L$. Conversely every smooth map $\varphi: M \rightarrow C P^{n}$ is obtained in this manner by considering the pull-back complex line bundle $\varphi^{-1} H$ over $M$ and a system of $n+1$ sections of $\varphi^{-1} H$ given by the homogeneous coordinates on $C P^{n}$. 
Hence giving a smooth map from $M$ to $C P^{n}$ is equivalent to giving a complex line bundle $L$ over $M$ and a system of $n+1$ smooth sections of $L$ with no common zeros.

Let $\varphi, \psi: M \rightarrow C P^{n}$ be two smooth maps. If $2 n \geqq \operatorname{dim} M$, then by the classification theorem we have

$$
\varphi \simeq \psi \Longleftrightarrow \varphi^{-1} H \equiv \psi^{-1} H,
$$

where $\varphi \simeq \psi$ means that $\varphi$ is homotopic to $\psi$. Since an equivalence class of complex line bundles is determined by the first Chern class, we get

$$
\begin{aligned}
\varphi^{-1} H \equiv \psi^{-1} H & \Longleftrightarrow c_{1}\left(\varphi^{-1} H\right)=c_{1}\left(\psi^{-1} H\right) \\
& \Longleftrightarrow \phi^{*} c_{1}(H)=\psi^{*} c_{1}(H) .
\end{aligned}
$$

Hence the homotopy class of a smooth map $\varphi: M \rightarrow C P^{n}$ is determined by the first Chern class $c_{1}(L)$ of the complex line bundle $L=\varphi^{-1} H$. When $M$ is oriented and $H^{2}(M, Z)=Z$, the degree of a map $\varphi: M \rightarrow C P^{n}$ is an integer $\operatorname{deg} \varphi$ defined by $c_{1}(L)=\varphi^{*} c_{1}(H)=(\operatorname{deg} \varphi) \omega_{1}$, where $\omega_{1}$ denotes a positive generator of $H^{2}(M, Z)$. Then we get

$$
\varphi \simeq \psi \Longleftrightarrow \operatorname{deg} \varphi=\operatorname{deg} \psi .
$$

Any two maps from $M$ to $C P^{n}$ constructed from systems of sections of the same line bundle are homotopic to each other if $2 n \geqq \operatorname{dim} M$. It is an interesting and important problem to find many harmonic maps in a given homotopy class of maps. We shall describe the harmonic map equation for a map $\phi$ in terms of $\tilde{\varphi}$.

Assume that $M$ is an $m$-dimensional Riemannian manifold. Denote by $\nabla^{M}$ the Riemannian connection of $M$. We endow the principal bundle $P$ with a connection $\Gamma$. The connection $\Gamma$ induces the covariant differentiation $\nabla^{L}$ in the associated line bundle $L$. For $X, Y \in C^{\infty}\left(T M^{c}\right)$, we denote by $X^{*}, Y^{*} \in C^{\infty}\left(T P^{c}\right)$ the horizontal lifts of $X, Y$ to $P$ with respect to the connection $\Gamma$. Let $\Phi$ be the universal lift of the map $\varphi$. By (1.1) we have

$$
\begin{aligned}
h\left((d \varphi)^{(1,0)}(X)\right) \widetilde{\varphi} & =\left(D_{X} \Phi\right) \widetilde{\varphi} \\
& =X^{*} \widetilde{\varphi}-\frac{\left\langle X^{*} \widetilde{\varphi}, \widetilde{\varphi}\right\rangle}{\langle\widetilde{\varphi}, \widetilde{\varphi}\rangle} \\
& =j\left(X^{*} \widetilde{\varphi}\right) .
\end{aligned}
$$

Here we regard $\widetilde{\varphi}$ as a section of the pull-back bundle $\varphi^{-1} H$. By a straightforward computation we get 


$$
\begin{aligned}
& h\left(\left(\nabla_{Y}(d \varphi)^{(1,0)}\right) X\right) \widetilde{\varphi} \\
& \quad=j\left(Y^{*} X^{*} \widetilde{\Phi}-\left(\nabla_{Y}^{M} X\right)^{*} \widetilde{\Phi}-\frac{\left\langle X^{*} \widetilde{\varphi}, \widetilde{\varphi}\right\rangle}{\langle\widetilde{\varphi}, \widetilde{\varphi}\rangle} Y^{*} \widetilde{\Phi}-\frac{\left\langle Y^{*} \widetilde{\varphi}, \widetilde{\varphi}\right\rangle}{\langle\widetilde{\varphi}, \widetilde{\varphi}\rangle} X^{*} \widetilde{\Phi}\right) .
\end{aligned}
$$

We denote by $\tau^{(1,0)} \in C^{\infty}\left(\varphi^{-1} T^{(1,0)} C P^{n}\right)$ the $(1,0)$-component of the tension field $\tau$ for the map $\varphi$. Then we have

$$
\begin{aligned}
h\left(\tau^{(1,0)}\right) \widetilde{\varphi} & =h\left(\sum_{i=1}^{m}\left(\nabla_{e_{i}}(d \varphi)^{(1,0)}\right)\left(e_{i}\right)\right) \widetilde{\Phi} \\
& =j\left(\sum_{i=1}^{m}\left(e_{i}^{*} e_{i}^{*} \widetilde{\Phi}-\left(\nabla_{e_{i}}^{M} e_{i}\right)^{*} \widetilde{\Phi}\right)-2 \sum_{i=1}^{m} \frac{\left\langle d \widetilde{\varphi}\left(e_{i}^{*}\right), \widetilde{\Phi}\right\rangle}{\langle\widetilde{\Phi}, \widetilde{\varphi}\rangle} d \widetilde{\Phi}\left(e_{i}^{*}\right)\right),
\end{aligned}
$$

where $\left\{e_{i}\right\}$ denotes a local orthonormal frame field on $M$. By virtue of $[\mathrm{K}-\mathrm{N}, \mathrm{I}, \mathrm{p} .115]$ or $[\mathrm{Oh} 1, \mathrm{p} .162]$, this equation becomes

$$
h\left(\tau^{(1,0)}\right) \tilde{\varphi}=j\left(-\left(\Delta^{L} \varphi\right)^{\sim}-2 \sum_{i=1}^{m} \frac{\left\langle\left(\nabla_{e_{i}}^{L} \varphi\right)^{\sim}, \tilde{\varphi}\right\rangle}{\langle\tilde{\varphi}, \tilde{\varphi}\rangle}\left(\nabla_{e_{i}}^{L} \varphi\right)^{\sim}\right),
$$

where $\varphi=\left(\varphi_{0}, \cdots, \varphi_{n}\right)$ and $\Delta^{L}=\left(\nabla^{L}\right)^{*} \nabla^{L}=-\sum_{i=1}^{m}\left(\nabla_{e_{i}}^{L} \nabla_{e_{i}}^{L}-\nabla_{\nabla_{e_{i} B_{i}}}^{L}\right)$.

PROPOSITION 1.1. (i) $\varphi$ is a harmonic map if and only if the system $\left\{\varphi_{0}, \cdots, \varphi_{n}\right\}$ satisfies

$$
\left(\Delta^{L} \varphi\right)^{\sim}+2 \sum_{i=1}^{m} \frac{\left\langle\left(\nabla_{i}^{L} \varphi\right)^{\sim}, \widetilde{\varphi}\right\rangle}{\langle\widetilde{\varphi}, \widetilde{\varphi}\rangle}\left(\nabla_{e_{i}}^{L} \varphi\right)^{\sim}=\mu \widetilde{\varphi}
$$

for some function $\mu$ on $M$.

(ii) Assume that $\Delta^{L} \varphi_{j}=\mu \varphi_{j}(j=0, \cdots, n)$ for some function $\mu$ on $M$. If $\tilde{\varphi}: P \rightarrow C^{n+1} \backslash\{0\}$ maps horizontal subspaces on $P$ to horizontal subspaces on $C^{n+1} \backslash\{0\}$, then $\varphi$ is a harmonic map.

Note that when $M$ is a complex manifold, $\varphi$ is a holomorphic map if and only if $D_{\bar{z}} \Phi=0$ for each $Z \in T^{(1,0)} M$.

Let $\Omega$ be the curvature form of the connection $\Gamma$ and $R^{L}$ be the curvature form of the connection $\nabla^{L}$ in the line bundle $L$. Then we have $\left(R^{L}\right)^{\sim}=\sigma(\Omega)$. The 2-form $\gamma_{1}(L)$ on $M$ defined by $\left(\gamma_{1}(L)\right)^{\sim}=$ $(V \overline{-1} / 2 \pi)\left(R^{L}\right)^{\sim}=(V \overline{-1} / 2 \pi) \sigma(\Omega)$ represents the first Chern class $c_{1}(L)$ of the line bundle $L$.

A smooth map $\varphi: M \rightarrow C P^{n}$ is called totally real if $\varphi$ satisfies $\varphi^{*} \omega=0$, where $\omega$ denotes the Kähler form of $C P^{n}$.

Proposition 1.2. If $\varphi: M \rightarrow C P^{n}(c)$ is a totally real map from a simply connected manifold $M$, then $\varphi$ has a horizontal lift $\widetilde{\varphi}: M \rightarrow$ $S^{2 n+1}(c / 4)$ relative to the Riemannian submersion $\pi: S^{2 n+1}(c / 4) \rightarrow C P^{n}(c)$. 
Moreover in case $M$ is a Riemannian manifold, $\phi$ is harmonic if and only if $\tilde{\phi}$ is harmonic.

Proof. The condition $\varphi^{*} \omega=0$ is equivalent to the flatness of the connection induced from the canonical connection of the principal bundle $\left(S^{2 n+1}, \pi, C P^{n}, S^{1}\right)$ through $\varphi$.

q.e.d.

§2. Homogeneous line bundles and homogeneous maps into complex projective spaces.

Let $M=G / K$ be an $m$-dimensional compact homogeneous space with a compact connected Lie group $G$. We denote by $g$ and the Lie algebras of $G$ and $K$, respectively. Let (, ) denote an $\operatorname{Ad}(G)$-invariant inner product of $g$ and $m$ the orthogonal complement of $\mathfrak{i n}$ ith respect to (, ). Let Aut $\left(C P^{n}\right)$ be the group of all holomorphic isometries of $C P^{n}$. $\operatorname{Aut}(\boldsymbol{C P})$ is identified in a natural way with a projective unitary group $P U(n+1)=S U(n+1) /\left\{\varepsilon 1_{n+1} ; \varepsilon^{n+1}=1\right\}$. For a given Lie group homomorphism $\rho: G \rightarrow \operatorname{Aut}\left(\boldsymbol{C} P^{n}\right)$, a map $\varphi: M \rightarrow C P^{n}$ is called $\rho$-equivariant if $\phi$ satisfies $\rho(a) \circ \varphi=\varphi \circ \gamma_{a}$ for each $a \in G$, where $\gamma_{a}$ denotes the natural action of $G$ on $M$. A map $\varphi: M \rightarrow C P^{n}$ is called G-equivariant if there exists a Lie group homomorphism $\rho: G \rightarrow \operatorname{Aut}\left(C P^{n}\right)$ such that $\varphi$ is $\rho$-equivariant.

In this section we consider the case when $M$ is a compact homogeneous space $G / K$ of a compact connected Lie group $G$ and the principal bundle $(P, \pi, M, K)$ is the standard principal bundle $(G, \pi, M, K)$ of the homogeneous space $M=G / K$. We endow $M$ with a $G$-invariant Riemannian metric $g$ on $M$. Suppose that $(\sigma, C)$ is a complex 1-dimensional unitary representation of $K$. Then the associated complex line bundle $L=G \times{ }_{\sigma} C$ becomes a $G$-homogeneous vector bundle with a Hermitian fibre metric $\langle,\rangle . C^{\infty}(L)$ and $C^{\infty}(G, C)_{K}$ have (left) $G$-module structures in a standard manner so that the map $A$ preserves the actions of $G$. Let $\mathscr{D}(G)$ denote the set of all equivalence classes of finite dimensional irreducible complex representations of $G$. Let for each $\Lambda \in \mathscr{D}(G),\left(\rho_{A}, V_{A}\right)$ be a fixed representation of $\Lambda$. For each $\Lambda \in \mathscr{D}(G)$ we assign a map $A_{\Lambda}$ from $V_{\Lambda} \otimes \operatorname{Hom}_{K}\left(V_{\Lambda}, C\right)$ to $C^{\infty}(G, C)_{K}$ by the rule $A_{\Lambda}(v \otimes T)(a)=T\left(\rho_{A}\left(a^{-1}\right) v\right)$. Here $\operatorname{Hom}_{K}\left(V_{A}, C\right)$ denotes the space of all linear maps $T$ of $V_{A}$ into $C$ such that $\sigma(k) \circ T=$ $T \circ \rho_{\Lambda}(k)$ for each $k \in K$. For $\Lambda \in \mathscr{D}(G)$, set $C_{A}^{\infty}(L)=A^{-1}\left(A_{A}\left(V_{\Lambda} \otimes \operatorname{Hom}_{K}\left(V_{A}\right.\right.\right.$, $C))$ ). By virtue of the Peter-Weyl theorem and the Frobenius reciprocity law, the algebraic direct sum $\sum_{\Lambda \in \mathscr{Q}(a)} C_{A}^{\infty}(L)$ is uniformly dense in $C^{\infty}(L)$ relative to the uniform topology (cf. [Wa 2]):

$$
C^{\infty}(L)=\sum_{\Lambda \in \mathscr{O}(Q)} C_{A}^{\infty}(L) .
$$


Choose the canonical $G$-invariant connection $\Gamma$ on the principal bundle $(G, \pi, M, K)$ with the horizontal subspaces determined by the subspace m. Let $\nabla^{L}$ be the covariant differentiation on the complex line bundle $L$ induced from the connection $\Gamma$. The Laplacian $\Delta^{L}$ gives the eigenspace decomposition of $C^{\infty}(L)$ in the same sense as (2.1):

$$
C^{\infty}(L)=\sum_{\nu} \mathscr{E}_{\nu}(L),
$$

where $\mathscr{E}_{2}(L)=\left\{f \in C^{\infty}(L) ; \Delta^{L} f=\nu f\right\}$ is the eigenspace of $\Delta^{L}$ corresponding to the eigenvalue $\nu$. Since $\Delta^{L}$ commutes with the action of $G$ on $C^{\infty}(L)$, each $\mathscr{E}_{2}(L)$ is a finite dimensional $G$-submodule. Hence each $\mathscr{E}_{\nu}(L)$ is a finite direct sum of some irreducible $G$-modules. Assume given a finite dimensional $G$-submodule $V$ of $C^{\infty}(L)$. Set $n+1=\operatorname{dim}_{c} V$. Decompose the $G$-module $V$ into the direct sum: $V=\sum_{i=1}^{s} V_{i}$ of irreducible $G$-modules $V_{i}$ with $\operatorname{dim}_{c} V_{i}=n_{i}+1$. For any real numbers $\left(r_{1}, \cdots, r_{s}\right)$ with $\sum_{i=1}^{i} r_{i}^{2}=1$, we can construct a smooth map $\varphi\left(V ; r_{1}, \cdots, r_{s}\right): M \rightarrow C P^{n}$ as follows. Choose a unitary basis $\left\{\varphi_{0}, \cdots, \varphi_{n}\right\}$ of $V$ with respect to the $L^{2}$-inner product such that $\left\{\varphi_{t(i-1)}, \cdots, \varphi_{t(i)-1}\right\}$ is a unitary basis of $V_{i}$, where $t(i)=\sum_{j=1}^{i} \operatorname{dim}_{c} V_{j}$ and $t(0)=0$. By the $G$-invariance of $\langle$,$\rangle and each$ $V_{i}$,

$$
\sum_{i=1}^{s} r_{i}^{2}\left(\left\langle\varphi_{t(i-1)}, \varphi_{t(i-1)}\right\rangle+\cdots+\left\langle\varphi_{t(i)-1}, \varphi_{t(i)-1}\right\rangle\right)
$$

is a positive constant on $M$. In particular the system

$$
\left\{r_{i} \varphi_{t(i-1)}, \cdots, r_{i} \varphi_{t(i)-1} ; i=1, \cdots, s\right\}
$$

has no common zeros. By using this system of sections of $L$, we obtain maps $\widetilde{\phi}\left(V ; r_{1}, \cdots, r_{s}\right): G \rightarrow C^{n+1} \backslash\{0\}$ and $\varphi\left(V ; r_{1}, \cdots, r_{s}\right) ; M \rightarrow C P^{n}$. Note that the $\operatorname{map} \varphi\left(V ; r_{1}, \cdots, r_{s}\right)$ is full.

We define a unitary representation $\rho_{v}: G \rightarrow U(n+1)$ by $L_{a}\left(\widetilde{\varphi}_{0}, \cdots, \widetilde{\varphi}_{n}\right)=$ $\left(\widetilde{\varphi}_{0}, \cdots, \widetilde{\varphi}_{n}\right) \rho_{V}(a)$ for each $a \in G$, where $L_{a}$ is the left action on $C^{\infty}(G, C)_{K}$. Note that $\rho_{V}(a) \in U\left(n_{1}+1\right) \times \cdots \times U\left(n_{s}+1\right)$. Then the map $\varphi\left(V ; r_{1}, \cdots, r_{s}\right)$ is $\rho_{V}$-equivariant for each $r_{1}, \cdots, r_{s}$ with $\sum_{i=1}^{s} r_{i}^{2}=1$. Hence we have

$$
\begin{aligned}
& \widetilde{\Phi}\left(V ; r_{1}, \cdots, r_{s}\right)(a)=\left(\rho_{V}(a)\right) v_{0}, \\
& \varphi\left(V ; r_{1}, \cdots, r_{s}\right)(a \cdot o)=\pi\left(\left(\rho_{V}(a)\right) v_{0}\right)
\end{aligned}
$$

for each $a \in G$. Here $o=e K \in M$ and $v_{0}=\widetilde{\phi}\left(V ; r_{1}, \cdots, r_{s}\right)(e) \in C^{n+1} \backslash\{0\}$.

Under the identification $\mathfrak{m} \cong T_{0} M$, choose an orthonormal basis $\left\{X_{1}, \cdots, X_{m}\right\}$ of $\mathfrak{m}$ with respect to $g$. By Proposition 1.1 the tension field $\tau$ of $\varphi\left(V ; r_{1}, \cdots, r_{s}\right)$ at $o \in M$ is given by 


$$
h\left(\tau^{(1,0)}\right) v_{0}=j\left(\left(\sum_{i=1}^{m} \rho_{V}\left(X_{i}\right)^{2}\right) v_{0}-2 \sum_{i=1}^{m}-\frac{\left\langle\rho_{V}\left(X_{i}\right) v_{0}, v_{0}\right\rangle}{\left\langle v_{0}, v_{0}\right\rangle} \rho_{V}\left(X_{i}\right) v_{0}\right)
$$

We describe the first Chern class $c_{1}(L)$ of the homogeneous line bundle $L$. The curvature form $\Omega$ of $\Gamma$ is given by

$$
\Omega(X, Y)=-[X, Y]_{\mathfrak{l}}
$$

for $X, Y \in \mathfrak{m}$ (cf. [K-N, I, p. 103]). We choose an element $\lambda_{\sigma} \in \mathfrak{l}$ such that $\sigma(X) v=\sqrt{-1}\left(\lambda_{\sigma}, X\right) v$ for any $X \in \mathfrak{l}$ and $v \in C$. Then the 2-form

$$
\left(\gamma_{1}(L)\right)^{\sim}(X, Y)=\frac{1}{2 \pi}\left(\left[\lambda_{a}, X\right], Y\right) \quad \text { for } \quad X, Y \in \mathfrak{m}
$$

represents the first Chern class $c_{1}(L)$ of $L$.

Assume that $(G, K)$ is a compact symmetric pair. Let $\mathfrak{g}=\mathfrak{t}+\mathfrak{m}$ be the canonical decomposition of $\mathfrak{g}$ associated with the symmetric pair $(G, K)$. Let a be a maximal abelian subspace of $\mathfrak{m}$. Choose a maximal abelian subalgebra $\mathfrak{h}$ of $\mathfrak{g}$ containing $\mathfrak{a}$. Fix a linear order $<$ on $\mathfrak{h}$. Let $\Delta(\subset \mathfrak{h})$ denote the root system of $\mathfrak{g}$ relative to $\mathfrak{h}$. We have the root space decomposition of $\mathfrak{g}^{c}$ relative to $\mathfrak{h}$ :

$$
\mathfrak{g}^{c}=\mathfrak{h}^{c}+\sum_{\alpha \in \Delta} \tilde{\mathfrak{g}}_{\alpha}
$$

where $\tilde{\mathfrak{g}}_{\alpha}=\left\{X \in \mathfrak{g}^{c} ;(\operatorname{ad} H) X=\sqrt{-1}(\alpha, H) X\right.$ for any $\left.H \in \mathfrak{h}\right\}$. Let $\Delta_{a}^{+}$be the set of positive roots which do not vanish identically on a. Set $\mathfrak{n}^{c}=$ $\sum_{\alpha \in \Delta_{a}^{+}} \widetilde{\mathfrak{g}}_{\alpha^{\circ}}$. Then $\mathfrak{n}$ is a nilpotent Lie algebra and the Iwasawa decomposition gives a direct sum decomposition

$$
\mathfrak{g}^{c}=\mathfrak{l}^{c}+\mathfrak{a}^{c}+\mathfrak{n}^{c}
$$

as vector spaces.

LEMMA 2.1. Let $\rho: G \rightarrow G L(V)$ be a complex irreducible representation of $G$ with the highest weight $\xi \in \mathfrak{h}$ and $\langle$,$\rangle be a G-invariant Hermitian$ inner product of $V$. Choose a weight vector $v_{\xi}(\neq 0) \in V$ for the highest weight $\xi$. Suppose that there exists a nonzero vector $w \in V$ and an element $\lambda \in \mathfrak{l}$ such that $\rho(X) w=V \overline{-1}(\lambda, X) w$ for each $X \in \mathfrak{t}$. Then we have $\left\langle w, v_{\xi}\right\rangle \neq 0$.

Proof. We define a complex valued linear function $F$ by $F(X)=$ $\left\langle\rho(X) v_{\xi}, w\right\rangle$ for $X \in \mathfrak{g}^{c}$. For any $Y \in \mathfrak{n}^{c}$, we have $F(Y)=0$ because $\rho(Y) v_{\xi}=0$. For any $H \in \mathfrak{a}^{c}$, we have $F(H)=V \overline{-1}(\xi, H)\left\langle v_{\xi}, w\right\rangle$. For any $X \in \mathfrak{l}$, we have $F(X)=\left\langle\rho(X) v_{\xi}, w\right\rangle=-\left\langle v_{\xi}, \rho(X) w\right\rangle=\sqrt{-1}(\lambda, X)\left\langle v_{\xi}, w\right\rangle$. If 
$\left\langle v_{\xi}, w\right\rangle=0$, then by (2.6) we get $F \equiv 0$. By the irreducibility of $\rho$, we have $w=0$, a contradiction.

q.e.d.

Proposition 2.2. Let $(G, K)$ be a compact symmetric pair and $\rho: G \rightarrow G L(V)$ be a complex irreducible representation of $G$. For any $\lambda \in \mathfrak{t}$, set

$$
W_{\lambda}=\{w \in V ; \rho(X) w=\sqrt{-1}(\lambda, X) w \text { for each } X \in \mathfrak{l}\} .
$$

Then we have $\operatorname{dim}_{c} W_{\lambda}=0$ or 1 .

Proof. Let $v_{\xi}$ denote the highest weight vector of $\rho$ as in Lemma 2.1 and $\langle$,$\rangle be a G$-invariant inner product of $V$. We define a linear map $f: W_{\lambda} \rightarrow C$ by $f(w)=\left\langle w, v_{\xi}\right\rangle$ for $w \in W_{\lambda}$. By Lemma 2.1, $f$ is injective. Therefore we have $\operatorname{dim}_{c} W_{2}=0$ or 1 .

q.e.d.

COROLlaRY 2.3. If $(G, K)$ is a compact symmetric pair, then in the decomposition (2.1) we have $C_{A}^{\infty}(L)$ is isomorphic to $V_{A}$ or $\{0\}$ for each $\Lambda \in \mathscr{D}(G)$.

REMARK. These results for $\lambda=0$ are well-known and essential in the theory of spherical functions over compact symmetric spaces. The proof here was inspired by that of [Te1].

\section{§3. Harmonicity of homogeneous maps to a complex projective space.}

Let $\varphi: M=G / K \rightarrow C P^{n}$ be a full, homogeneous map compatible with a Lie group homomorphism $\rho: G \rightarrow \operatorname{Aut}\left(C P^{n}\right)$. Note that there exists a unitary representation $\tilde{\rho}: \widetilde{G} \rightarrow S U(n+1)$ of the finite covering group $\widetilde{G}$ of $G$ such that the diagram

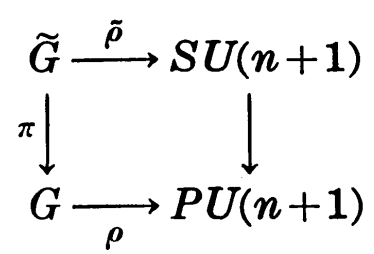

is commutative. Take $v_{0} \in S^{2 n+1}(c / 4)$ with $\varphi(o)=C v_{0}$. Then we have $\varphi(a \cdot o)=\rho(a) \varphi(o)=\rho(a) \pi\left(v_{0}\right)=\pi\left(\tilde{\rho}(\widetilde{a}) v_{0}\right)$ for each $\widetilde{a} \in \widetilde{G}$ with $\pi(\widetilde{a})=a \in G$. In particular we have $\tilde{\rho}(\widetilde{K}) \boldsymbol{C} v_{0} \subset \boldsymbol{C} v_{0}$. Hence there is a real-valued linear form $\lambda_{0}$ on $\mathfrak{t}$ such that $\tilde{\rho}(X) v_{0}=\sqrt{-1} \lambda_{0}(X) v_{0}$ for any $X \in \mathfrak{t}$. Set $\mathfrak{t}^{\prime}=\operatorname{Ker} \lambda_{0}=$ $\left\{X \in \mathfrak{t} ; \tilde{\rho}(X) v_{0}=0\right\}$. We have a decomposition $\mathfrak{t}=\mathfrak{t}^{\prime} \oplus \mathfrak{c}_{0}$ as Lie algebras, where $\mathfrak{c}_{0}$ is an orthogonal complement of $\mathfrak{t}^{\prime}$ in $\mathfrak{t}$. Note that $\operatorname{dim} \mathfrak{c}_{0}=0$ or 
1 and $c_{0}$ is contained in the center of $k$.

Decompose the representation space $C^{n+1}$ of $\tilde{\rho}$ into the direct sum:

$$
C^{n+1}=\sum_{i=1}^{8} V_{i}
$$

of irreducible $\widetilde{G}$-submodules $V_{i}$, and let

$$
v_{0}=\sum_{i=1}^{8} v_{i}, \quad v_{i} \in V_{i}(1 \leqq i \leqq 8)
$$

Then each $v_{i}$ does not vanish in virtue of the fullness of $\varphi$, and satisfies

$$
\tilde{\rho}(X) v_{i}=V \overline{-1} \lambda_{0}(X) v_{i} \quad \text { for each } X \in \mathfrak{i} .
$$

Put $W=\boldsymbol{C} v_{0}$. Then $W$ is a complex 1-dimensional $\widetilde{K}$-submodule of $C^{n+1}$. Consider the associated homogeneous complex line bundle $L=$ $\widetilde{G} \times{ }_{\sigma^{*}} W^{*}$ over $M=\widetilde{G} / \widetilde{K}$, where $\left(\sigma^{*}, W^{*}\right)$ denotes the dual $\widetilde{K}$-module of $W$. We define a smooth map $\widetilde{\varphi}=\left(\widetilde{\varphi}_{0}, \cdots, \widetilde{\varphi}_{n}\right): \widetilde{G} \rightarrow\left(W^{*}\right)^{n+1} \approx C^{n+1}$ by $\left(\widetilde{\varphi}_{\imath}(a)\right)(w)=$ $\left\langle\tilde{\rho}(a) w, \varepsilon_{i}\right\rangle(i=0, \cdots, n)$ for each $a \in \widetilde{G}$ and $w \in W$. Since each $\widetilde{\varphi}_{i}$ satisfies $\widetilde{\varphi}_{i}(a k)=\sigma(k)^{-1} \widetilde{\varphi}_{i}(a)$, i.e. $\widetilde{\varphi}_{i} \in C^{\infty}\left(\widetilde{G}, W^{*}\right)_{\tilde{K}},\left\{\widetilde{\varphi}_{0}, \cdots, \widetilde{\varphi}_{n}\right\}$ induces a system $\left\{\varphi_{0}, \cdots, \varphi_{n}\right\}$ of smooth sections of the bundle $L$. Let $V$ denote the $\widetilde{G}-$ submodule of $C^{\infty}(L)$ spanned by $\varphi_{0}, \cdots, \varphi_{n}$. Then $\phi$ is equivalent to $\varphi\left(V ; r_{1}, \cdots, r_{s}\right)$ for some real numbers satisfying $\sum_{i=1}^{i} r_{i}^{2}=1$.

We shall study harmonicity of the homogeneous map $\varphi=\varphi\left(V ; r_{1}, \cdots, r_{s}\right)$. We prepare a lemma. Choose a subspace $\mathfrak{m}$ of $\mathfrak{g}$ such that $\operatorname{Ad}(K) \mathfrak{m}=\mathfrak{m}$ and $\mathfrak{g}=\mathfrak{t}+\mathfrak{m}$ is a direct sum as vector subspaces.

LEMMA 3.1. $\tilde{\rho}([\mathfrak{t}, \mathfrak{m}]) v_{0}$ is horizontal with respect to the Hopf fibration $\pi: S^{2 n+1}(c / 4) \rightarrow C P^{n}$, that is, $\left\langle\tilde{\rho}([\mathfrak{t}, \mathfrak{m}]) v_{0}, v_{0}\right\rangle=0$. Therefore if $[\mathfrak{t}, \mathfrak{m}]=\mathfrak{m}$, then $\tilde{\rho}(\mathfrak{m}) v_{0}$ is horizontal. Here $[\mathfrak{l}, \mathfrak{m}]$ denotes the vector subspace of $\mathfrak{m}$ spanned by $\{[T, X] ; T \in \mathfrak{k}, X \in \mathfrak{m}\}$.

Proof. From the invariance of the Hermitian inner product $\langle$, by the action $\tilde{\rho}$ of $\widetilde{G}$, for any $T \in \mathfrak{l}$ and $X \in \mathfrak{m}$ we have

$$
\left\langle\tilde{\rho}(T) \tilde{\rho}(X) v_{0}, v_{0}\right\rangle+\left\langle\tilde{\rho}(X) v_{0}, \tilde{\rho}(T) v_{0}\right\rangle=0 \text {. }
$$

Since $\tilde{\rho}(T) v_{0}=\sqrt{-1} \lambda_{0}(T) v_{0}$, we have

$$
\left\langle\tilde{\rho}(X) v_{0}, \tilde{\rho}(T) v_{0}\right\rangle=-\sqrt{-1} \lambda_{0}(T)\left\langle\tilde{\rho}(X) v_{0}, v_{0}\right\rangle,
$$

and

$$
\begin{aligned}
\left\langle\tilde{\rho}(T) \tilde{\rho}(X) v_{0}, v_{0}\right\rangle & =\left\langle\tilde{\rho}([T, X]) v_{0}, v_{0}\right\rangle+\left\langle\tilde{\rho}(X) \tilde{\rho}(T) v_{0}, v_{0}\right\rangle \\
& =\left\langle\tilde{\rho}([T, X]) v_{0}, v_{0}\right\rangle+\sqrt{-1} \lambda_{0}(T)\left\langle\tilde{\rho}(X) v_{0}, v_{0}\right\rangle
\end{aligned}
$$


Hence we obtain $\left\langle\tilde{\rho}([T, X]) v_{0}, v_{0}\right\rangle=0$.

q.e.d.

REMARK. Set $\mathfrak{m}=[\mathfrak{t}, \mathfrak{m}]+\mathfrak{m}_{0}$ where $\mathfrak{m}_{0}$ denotes the orthogonal complement of $[\mathfrak{t}, \mathfrak{m}]$ in $\mathfrak{m}$ with respect to an $\operatorname{Ad}(G)$-invariant inner product of $\mathfrak{g}$. Then $\mathfrak{m}_{0}$ is $\operatorname{Ad}(K)$-invariant and satisfies $\left[\mathfrak{l}, \mathfrak{m}_{0}\right]=0$ and $\left[\mathfrak{m}, \mathfrak{m}_{0}\right] \subset \mathfrak{m}$.

If the homogeneous space $M=G / K$ satisfies one of the following conditions, then we have $\mathfrak{m}=[\mathfrak{l}, \mathfrak{m}]$ :

(1) The isotropy representation of $M=G / K$ is irreducible.

(2) $\operatorname{rank} G=\operatorname{rank} K$.

(3) $\mathfrak{g}$ is semisimple and $(\mathfrak{g}, \mathfrak{t})$ is a symmetric Lie algebra.

The compact homogeneous spaces with irreducible isotropy representation were classified by [Wo]. By a result of Hopf-Samelson $M=G / K$ satisfies $\operatorname{rank} G=\operatorname{rank} K$ if and only if the Euler-Poincaré characteristic of $M$ is positive.

Let $g$ be a $G$-invariant Riemannian metric on $M$. Let $g_{\theta}$ denote the $G$-invariant Riemannian metric on $M$ induced by an $\operatorname{Ad}(G)$-invariant inner product (, ) of $g$.

From Proposition 1.1, (2.3) and Lemma 3.1 we get the following.

Proposition 3.2. Suppose that the homogeneous space $M=G / K$ satisfies the condition $[\mathfrak{t}, \mathfrak{m}]=\mathfrak{m}$. Then the following statements for a $G$-equivariant map $\varphi=\varphi\left(V ; r_{1}, \cdots, r_{s}\right):(M, g) \rightarrow C P^{n}$ are equivalent to each other:

(1) $\varphi$ is a harmonic map.

(2) $\left(\sum_{i=1}^{m} \tilde{\rho}\left(X_{i}\right)^{2}\right) v_{0} \in R v_{0}$, where $\left\{X_{1}, \cdots, X_{m}\right\}$ denotes an orthonormal basis of $\mathfrak{m}$ with respect to $g$.

(3) For some constant $\nu, \Delta^{L} \varphi_{j}=\nu \varphi_{j}(j=0, \cdots, n)$, that is, the subspace $V$ of $C^{\infty}(L)$ is contained in some eigenspace $\mathscr{E}_{\nu}(L)$ of $\Delta^{L}$.

By this proposition we see that if $M=G / K$ satisfies $[\mathfrak{l}, \mathfrak{m}]=\mathfrak{m}$, then $\left\{\varphi\left(V ; r_{1}, \cdots, r_{s}\right) ; V\right.$ is a $\widetilde{G}$-submodule of some eigenspace for $\Delta^{L}, r_{i} \in \boldsymbol{R}$ $(i=1, \cdots, s)$ with $\left.\sum_{i=1}^{s} r_{i}^{2}=1\right\}$ is the set of all equivalence classes of $G$ equivariant harmonic maps from $(M=G / K, g)$ to a complex projective space. Here $s$ denotes the number of $\widetilde{G}$-irreducible components of $V$.

Let $\mathscr{C}$ denote the Casimir differential operator of $\widetilde{G}$ with respect to the $\operatorname{Ad}(G)$-invariant inner product $($,$) of \mathrm{g}$, that is, $\mathscr{C}=\sum_{A=1}^{l} X_{A} X_{A}$, where $\left\{X_{A}\right\}_{A=1, \cdots, l}$ is an orthonormal basis of $g$ with respect to $($,$) and$ $\left\{X_{k}\right\}_{k=m+1}, \cdots, l$ is contained in $\mathfrak{t}$. If $g=g_{G}$, then we have $\tilde{\rho}\left(\sum_{i=1}^{m} X_{i} X_{i}\right)=$ $\tilde{\rho}(\mathscr{C})-\sum_{k=m+1}^{l} \tilde{\rho}\left(X_{k}\right)^{2}$. If the representation $\left(\tilde{\rho}, C^{n+1}\right)$ is irreducible, then by Schur's lemma $\tilde{\rho}(\mathscr{C})$ is a constant operator $-c(\tilde{\rho}) I$ for some $c(\tilde{\rho})>0$. In this case we have $\tilde{\rho}\left(\sum_{i=1}^{m} X_{i} X_{i}\right) v_{0}=\left(-c(\tilde{\rho})+\left(\lambda_{0}, \lambda_{0}\right)\right) v_{0}$. Therefore we 
obtain the following.

Proposition 3.3. Suppose that $M=G / K$ satisfies the condition $[\mathfrak{t}, \mathfrak{m}]=\mathfrak{m}$. If $V$ is irreducible (equivalently, $\left(\tilde{\boldsymbol{\rho}}, \boldsymbol{C}^{n+1}\right)$ is irreducible), then $\varphi=\varphi\left(V ; r_{1}, \cdots, r_{s}\right):\left(M, g_{G}\right) \rightarrow C P^{n}$ is a harmonic map $(s=1)$.

We shall give an explicit formula for the energy density of a $G$ equivariant map $\varphi:\left(M, g_{G}\right) \rightarrow C P^{n}$.

Proposition 3.4. Suppose that $M=G / K$ satisfies the condition $[\mathfrak{f}, \mathfrak{m}]=\mathfrak{m}$.

(1) The energy density $e(\varphi)$ of a G-equivariant map $\varphi:\left(M, g_{G}\right) \rightarrow$ $\left(C P^{n}, g_{c}\right)$ is given by

$$
e(\varphi)=-\frac{1}{2}\left\langle\tilde{\rho}(\mathscr{C}) v_{0}, v_{0}\right\rangle-\frac{2\left|\lambda_{0}\right|^{2}}{c} .
$$

Moreover if $\left(\tilde{\rho}, C^{n+1}\right)$ is irreducible, then

$$
e(\varphi)=\frac{2}{c}\left(c(\tilde{\rho})-\left|\lambda_{0}\right|^{2}\right) .
$$

(2) If $\phi^{*} g_{c}=r g_{G}$ for some positive constant $r>0$, then $r$ is given by

$$
r=-\frac{1}{\operatorname{dim} M}\left\{\left\langle\tilde{\rho}(\mathscr{C}) v_{0}, v_{0}\right\rangle+\frac{4}{c}\left|\lambda_{0}\right|^{2}\right\} .
$$

Proof. Under the identification $\mathfrak{m} \cong T_{o} M$, by Lemma 3.1 we compute

$$
\begin{aligned}
e(\varphi) & =\frac{1}{2} \sum_{i=1}^{m}\left(\Phi^{*} g_{c}\right)\left(X_{i}, X_{i}\right) \\
& =\frac{1}{2} \sum_{i=1}^{m}\left\langle\tilde{\rho}\left(X_{i}\right) v_{0}, \tilde{\rho}\left(X_{i}\right) v_{0}\right\rangle \\
& =-\frac{1}{2}\left\langle\tilde{\rho}\left(\sum_{i=1}^{m} X_{i} X_{i}\right) v_{0}, v_{0}\right\rangle \\
& =-\frac{1}{2}\left(\left\langle\tilde{\rho}(\mathscr{C}) v_{0}, v_{0}\right\rangle+\sum_{k=m+1}^{l} \lambda_{0}\left(X_{k}\right)^{2}\left|v_{0}\right|^{2}\right) \\
& =-\frac{1}{2}\left\{\left\langle\tilde{\rho}(\mathscr{C}) v_{0}, v_{0}\right\rangle+\frac{4}{c}\left|\lambda_{0}\right|^{2}\right\} .
\end{aligned}
$$

From this we get (1). Immediately (1) implies (2). q.e.d.

By virtue of the formula of Freudenthal, the eigenvalue $c(\tilde{\rho})$ of the Casimir operator $\tilde{\rho}(\mathscr{C})$ for an irreducible representation $\left(\tilde{\rho}, C^{n+1}\right)$ is given by 


$$
c(\tilde{\rho})=\left(\Lambda_{\tilde{\rho}}, \Lambda_{\tilde{\rho}}+2 \delta\right),
$$

where $\Lambda_{\tilde{\rho}}$ denotes the highest weight of the representation $\tilde{\rho}$ and $\delta$ denotes half the sum of the positive roots of $\mathfrak{g}$, relative to a maximal abelian subalgebra of $\mathfrak{g}$ and a linear order on it.

REMARK. (1) Similar results and formulas can be found in [Gu 1], [Gu 3] for a flag manifold $G / T$. In [Gu 3], [Gu 4], Guest gives more results and interesting observations from the viewpoint of $t$ wistor geometry and symplectic geometry.

(2) If $K$ has the discrete center, then the associated homogeneous complex line bundle $L$ is a flat vector bundle and any homogeneous map $\varphi$ made from sections of $L$ is always totally real.

§4. Homogeneous harmonic maps of compact homogeneous Kähler manifolds into complex projective spaces.

Let $g$ be a compact semisimple Lie algebra and $t$ be a maximal abelian subalgebra of $g$. Denote by $g^{c}$ and $t^{c}$ the complexifications of $g$ and $t$, respectively. $\mathfrak{t}^{c}$ is a Cartan subalgebra of $\mathfrak{g}^{c}$. Let $($,$) be an \operatorname{Ad}(G)$ invariant inner product on $g$ defined by $(-1)$ times the Killing form of g. Let $\Sigma(\subset t)$ denote the root system of $g$ relative to $t$. We have a root space decomposition of $g^{c}$ :

$$
\mathrm{g}^{c}=\mathrm{t}^{c}+\sum_{\alpha \in \Sigma} g_{\alpha}^{c},
$$

where $\mathrm{g}_{\alpha}^{c}=\left\{X \in \mathrm{g}^{c} ;(\operatorname{ad} H) X=\sqrt{-1}(\alpha, H) X\right.$ for any $\left.H \in \mathfrak{t}\right\}$. Choose a lexicographic order $>$ on $\Sigma$. Put $\Sigma^{+}=\{\alpha \in \Sigma ; \alpha>0\}$. Let $\Pi$ be the fundamental root system of $\Sigma$ consisting of simple roots with respect to the linear order $>$. We identify $\Pi$ with its Dynkin diagram. Let $\left\{\Lambda_{\alpha}\right\}_{\alpha \in \Pi}(\subset t)$ be the fundamental weight system of $g^{c}$ corresponding to $\Pi$ :

$$
\frac{2\left(\Lambda_{\alpha}, \beta\right)}{(\beta, \beta)}=\left\{\begin{array}{lll}
1 & \text { if } & \alpha=\beta, \\
0 & \text { if } & \alpha \neq \beta
\end{array}\right.
$$

Let $\Pi_{0}$ be a subdiagram of $\Pi$. We may suppose that the pair $\left(\Pi, \Pi_{0}\right)$ is effective, that is, $\Pi_{0}$ contains no irreducible component of $\Pi$. Put $\Sigma_{0}=\Sigma \cap\left\{\Pi_{0}\right\}_{z}$, where $\left\{\Pi_{0}\right\}_{z}$ denote the subgroup of $t$ generated by $\Pi_{0}$ over $Z$. Define a subalgebra $\mathfrak{u}$ of $\mathfrak{g}^{c}$ by

$$
\mathfrak{u}=\mathfrak{t}^{c}+\sum_{\alpha \in \Sigma_{0} \cup \Sigma^{+}} \mathfrak{g}_{-\alpha}^{\boldsymbol{c}} .
$$

Let $G^{c}$ be the connected complex semisimple Lie group without 
center, whose Lie algebra is $\mathrm{g}^{c}$, and $U$ the connected closed complex subgroup of $G^{c}$ generated by $\mathfrak{u}$. We define a complex manifold $M$ by $M=G^{c} / U$, which is known to be compact and simply connected. Denote by $J$ the complex structure of $M . G^{c}$ acts effectively on $M$, since $G^{c}$ has no center. The origin $U$ of $M$ is denoted by $o$. Let $G$ be a compact connected semisimple subgroup of $G^{c}$ generated by $g$ and put $K=G \cap U$. Then $K$ is connected and $G$ acts on $M$ transitively, and hence the natural map $G / K \rightarrow G^{c} / U$ induces an identification $M=G / K$ as a smooth manifold. $M$ is called a generalized flag manifold. The complexification $\mathfrak{i}^{c}$ of the Lie algebra $\mathrm{t}$ of $K$ is given by

$$
\mathfrak{t}^{c}=\mathrm{t}^{c}+\sum_{\alpha \in \Sigma_{0}} \mathfrak{g}_{\alpha}^{c}
$$

We define a subspace $c$ of $t$ by

$$
\mathrm{c}=\sum_{\alpha \in \Pi-\Pi_{0}} \boldsymbol{R} \Lambda_{\alpha}
$$

Then c coincides with the center of $\mathfrak{t}$. We define lattices $Z$ and $Z_{\mathrm{c}}$ of $\mathfrak{t}$ and $c$ respectively by

$$
Z=\sum_{\alpha \in \Pi} Z \Lambda_{\alpha}
$$

and

$$
Z_{\mathrm{c}}=Z \cap \mathrm{c}=\sum_{\alpha \in \Pi-\Pi_{0}} Z \Lambda_{\alpha}
$$

We define a cone $c^{+}$in $c$ by

$$
\left.c^{+}\left(\text {resp. } c^{\sharp}\right)=\{\lambda \in c \backslash\{0\} ;(\lambda, \alpha)>0 \text { (resp. } \geqq 0) \text { for each } \alpha \in \Pi-\Pi_{0}\right\}
$$

and put $Z_{\mathrm{c}}^{+}=Z \cap \mathrm{c}^{+}$and $Z_{\mathrm{c}}^{*}=Z \cap \mathrm{c}^{*}$. Then we have $\mathrm{c}^{+}=\sum_{\alpha \in \Pi-\Pi_{0}} R^{+} \Lambda_{\alpha}$ and $Z_{c}^{+}=\sum_{\alpha \in \Pi-\Pi_{0}} Z^{+} \Lambda_{\alpha}$, where $R^{+}$and $Z^{+}$denote the sets of positive real numbers and positive integers, respectively. Let $\mathfrak{m}$ be the orthogonal complement of in $\mathfrak{g}$ with respect to $($,$) . Then we have a direct sum$ decomposition $\mathfrak{g}=\mathfrak{t}+\mathfrak{m}$ as vector spaces. The subspace $\mathfrak{m}$ is $K$-invariant under the adjoint action and identified with the tangent space $T_{o} M$ of $M$ at the origin. Put $\Sigma_{\mathrm{m}}^{+}=\Sigma^{+}-\Sigma_{0}, \Sigma_{\mathrm{m}}^{-}=-\Sigma_{\mathrm{m}}^{+}$, and define $K$-invariant subspaces $\mathfrak{m}^{ \pm}$of $\mathfrak{g}^{c}$ by

$$
\mathfrak{m}^{ \pm}=\sum_{\alpha \in \Sigma_{\mathfrak{m}}^{ \pm}} \mathfrak{g}_{\alpha}^{c}
$$

Then $\overline{\mathfrak{m}}^{ \pm}=\mathfrak{m}^{\mp}$ and the complexification $\mathfrak{m}^{c}$ of $\mathfrak{m}$ is the direct sum $\mathfrak{m}^{c}=$ $\mathfrak{m}^{+}+\mathfrak{m}^{-}$. 
Let $\widetilde{G}^{c}$ be the universal covering group of $G^{c}$ and $\widetilde{G}$ be the simply connected subgroup of $\widetilde{G}^{c}$ generated by $g$. Let $\widetilde{U}$ be the connected closed complex subgroup of $\widetilde{G}^{c}$ generated by $\mathfrak{u}$ and put $\widetilde{K}=\widetilde{G} \cap \widetilde{U}$. Then we have also identifications $M=\widetilde{G}^{c} / \widetilde{U}=\widetilde{G} / \widetilde{K}$. Let $\widetilde{T}$ be the toral subgroup of $\widetilde{G}$ generated by $\mathrm{t}$.

We choose $E_{\alpha} \in \mathfrak{g}_{\alpha}^{c}$ for $\alpha \in \Sigma$ with the following properties and fix them once and for all:

$$
\left[E_{\alpha}, E_{-\alpha}\right]=V \overline{-1} \alpha, \quad\left(E_{\alpha}, E_{-\alpha}\right)=1, \quad \bar{E}_{\alpha}=E_{-\alpha} \quad \text { for } \quad \alpha \in \Sigma .
$$

We denote by $X \mapsto \bar{X}$ the complex conjugation of $g^{c}$ with respect to the real form g. Let $\left\{\omega^{\alpha}\right\}_{\alpha \in \Sigma}$ be the linear forms on $g^{c}$ dual to $\left\{E_{\alpha}\right\}_{\alpha \in \Sigma}$, more precisely, the linear forms defined by

$$
\begin{aligned}
& \omega^{\alpha}\left(t^{c}\right)=\{0\}, \\
& \omega^{\alpha}\left(E_{\beta}\right)=\left\{\begin{array}{lll}
1 & \text { if } & \alpha=\beta, \\
0 & \text { if } & \alpha \neq \beta .
\end{array}\right.
\end{aligned}
$$

Every $G$-invariant Kähler metric on $M$ is given by

$$
g(\lambda)=\frac{1}{2 \pi} \sum_{\alpha \in \Sigma_{\mathrm{m}}^{+}}(\lambda, \alpha) \omega^{\alpha} \cdot \bar{\omega}^{\alpha} \quad \text { for } \lambda \in \mathrm{c}^{+} \quad \text { (cf. [B-H], [Te 2]). }
$$

Denoted by $\omega(\lambda)$ the Kähler form (or fundamental 2-form) of $g(\lambda)$, $\omega(\lambda)(X, Y)=g(\lambda)(J X, Y)$. Any $G$-invariant Riemannian metric on $M$ is a Hermitian metric with coclosed fundamental 2-form.

Given any $\lambda \in Z_{c}$, we can define a complex 1-dimensional unitary representation $\sigma(\lambda)$ of $\widetilde{K}$ by $(\sigma(\lambda))(a)=\exp (V \overline{-1}(\lambda, X))$ for each $a \in \widetilde{K}$, where $a=\exp X$ and $X \in \mathfrak{f}$. We construct a homogeneous complex line bundle $L_{\lambda}=\widetilde{G} \times_{\sigma(\lambda)} C$ over $M=\widetilde{G} / \widetilde{K}$ associated to the representation $(\sigma(\lambda), C)$ of $\widetilde{K}$. Conversely for each homogeneous complex line bundle $L$ over $M=\widetilde{G} / \widetilde{K}$, there exists a unique element $\lambda \in Z_{\mathrm{c}}$ such that $L=L_{\lambda}$. The first Chern class $c_{1}\left(L_{\lambda}\right)$ of the complex line bundle $L_{\lambda}$ over $M$ associated to $\lambda \in Z_{\mathrm{c}}$ is represented by the closed 2-form

$$
\gamma_{1}\left(L_{\lambda}\right)=\frac{\sqrt{-1}}{2 \pi} \sum_{\alpha \in \Sigma_{\mathrm{m}}^{ \pm}}(\lambda, \alpha) \omega^{\alpha} \wedge \bar{\omega}^{\alpha} .
$$

This is because by (2.5) we have

$$
\gamma_{1}\left(L_{\lambda}\right)\left(E_{\alpha}, \bar{E}_{\beta}\right)=\frac{1}{2 \pi}\left(\left[\lambda, E_{\alpha}\right], \bar{E}_{\beta}\right)=\frac{V \overline{-1}}{2 \pi}(\lambda, \alpha)\left(E_{\alpha}, \bar{E}_{\beta}\right) .
$$

Proposition 4.1 (cf. [B-H], [Te 2]). Let $\mathscr{F}_{a}^{2}(M)$ and $\mathscr{C}^{2}(M, g)$ be 
the real vector space of all G-invariant closed 2-forms on $M$ and the real vector space of all harmonic 2-forms on $M$ with respect to a $G$-invariant Riemannian metric $g$ on $M$, respectively. Then we have the following isomorphisms:

$$
\begin{aligned}
& \mathrm{c} \cong H^{1}(\widetilde{K}, \boldsymbol{R}) \cong H^{2}(M, \boldsymbol{R}) \cong \mathscr{Z}^{2}(M, g)=\mathscr{J}_{G}^{2}(M) \\
& \prod_{\mathrm{c}} \cong H^{1}(\widetilde{K}, \boldsymbol{Z}) \cong H^{2}(M, \boldsymbol{Z})
\end{aligned}
$$

and the linear isomorphism between $\mathrm{c}$ and $\mathscr{F}_{G}^{2}(M)$ is given by

$$
\eta(\lambda)=\frac{\sqrt{-1}}{2 \pi} \sum_{\alpha \in \Sigma_{\mathrm{m}}^{+}}(\lambda, \alpha) \omega^{\alpha} \wedge \bar{\omega}^{\alpha} \in \mathscr{I}_{G}^{2}(M)
$$

for $\lambda \in c$. Here note that we can identify $H^{1}(\widetilde{K}, Z)$ and $H^{2}(M, Z)$ with subgroups of $H^{1}(\widetilde{K}, \boldsymbol{R})$ and $H^{2}(M, R)$ since these integral cohomology groups have no torsion.

Let $\mathscr{L}(M)$ be the complete set of equivalence classes of complex line bundles over $M$. Then we get the bijective correspondences among $\mathscr{L}(M), H^{2}(M, Z)$ and $Z_{\text {c }}$ by $c_{1}\left(L_{\lambda}\right)=\eta(\lambda)$ for $\lambda \in Z$.

For each $\lambda \in Z_{\iota}$, there is a unique holomorphic character $\chi(\lambda)$ of $\widetilde{U}$ such that

$$
(\chi(\lambda))(\exp H)=\exp V \overline{-1}(\lambda, H) \quad \text { for each } H \in t^{c} .
$$

Then we can identify $L_{\lambda}$ with the holomorphic line bundle $\widetilde{G}^{c} \times_{\chi_{(\lambda)}} C$ over $M$ associated to the principal bundle $\left(\widetilde{G}^{c}, \pi, M, \widetilde{U}\right)$ by $\chi(\lambda)$. We have the identifications $L_{\lambda}=\widetilde{G} \times_{o(\lambda)} C=\widetilde{G}^{c} \times_{x(\lambda)} C$. Through these identifications we endow the complex line bundle $L_{\lambda}$ with the Hermitian metric and the holomorphic structure. Hence $L_{\lambda}$ becomes a holomorphic Hermitian line bundle. Let $\Gamma$ be the canonical $G$-invariant connection on the principal bundle $(G, \pi, M, K)$ and let $g=g(\mu)\left(\mu \in \mathrm{c}^{+}\right)$be a $G$-invariant Kähler metric on $M$. By (2.4) and (4.1) the curvature form $\Omega$ of $\Gamma$ is of type $(1,1)$. Let $\nabla^{\lambda}$ denote the covariant differentiation of $L_{\lambda}$ induced from the connection $\Gamma$. Then $\nabla^{\lambda}$ is the Hermitian connection of the holomorphic Hermitian line bundle $L_{\lambda}$. The complex Laplacian $\square^{2}$ of $L_{\lambda}$ is defined by

$$
\square^{\lambda} f=\bar{\partial} * \bar{\partial} f=-\sum_{i=1}^{m}\left[\nabla_{u_{i}}^{\lambda} \nabla_{\bar{u}_{i}}^{\lambda} f-\nabla_{\bar{v}_{i}}^{\lambda} \bar{u}_{i} f\right] \quad \text { for } f \in C^{\infty}\left(L_{\lambda}\right),
$$

where $\bar{\partial}$ and $\bar{\partial}^{*}$ denote the $(0,1)$-operator of the holomorphic line bundle $L_{\lambda}$ and its adjoint operator respectively, and $\left\{u_{i}\right\}$ is a unitary basis of 
$T_{p}^{(1,0)} M$ with respect to $g=g(\mu)$. Then we have

$$
\begin{aligned}
\square^{2} f & =-\sum_{i=1}^{m} \nabla_{u_{i}, \bar{u}_{i}}^{\lambda} f=-\frac{1}{2} \sum_{i=1}^{m}\left[\nabla_{u_{i}, \bar{u}_{i}}^{\lambda} f+\nabla_{\bar{u}_{i}, u_{i}}^{\lambda} f+R^{\lambda}\left(u_{i}, \bar{u}_{i}\right) f\right] \\
& =\frac{1}{2}\left\{\Delta^{2} f-2 \pi\left[\sum_{\alpha \in \Sigma_{i=1}^{+}} \frac{(\lambda, \alpha)}{(\mu, \alpha)}\right] f\right\},
\end{aligned}
$$

where $\Delta^{\lambda}$ and $R^{\lambda}$ denote the rough Laplacian relative to $\nabla^{2}$ and the curvature form of $\nabla^{2}$ respectively. Hence if $\lambda=\mu$, that is, $c_{1}\left(L_{2}\right)_{R}=[\omega(\mu)]$, then it becomes $\square^{2}=(1 / 2)\left(\Delta^{2}-2 \pi m \cdot 1\right)$, where $m=\operatorname{dim}_{c} M$. Set $\nu(\lambda, \mu)=$ $2 \pi \sum_{\alpha \in \Sigma_{\Sigma^{+}}}(\lambda, \alpha) /(\mu, \alpha)$. In the next section we will determine all eigenvalues and eigenspaces of the Laplacians $\square^{2}$ and $\Delta^{2}$ for each irreducible Hermitian symmetric space $M$ of compact type.

Let $\Gamma\left(L_{2}\right)$ be the subspace of $C^{\infty}\left(L_{2}\right)$ consisting of all holomorphic sections of $L_{\lambda}$. We see that $\Gamma\left(L_{\lambda}\right)=\left\{f \in C^{\infty}\left(L_{\lambda}\right) ; \square^{\lambda} f=0\right\}=\left\{f \in C^{\infty}\left(L_{\lambda}\right) ; \Delta^{\lambda} f=\right.$ $\nu(\lambda, \mu) f\}$. From the Borel-Weil theorem ([Bt]) we know that $\Gamma\left(L_{\lambda}\right)$ is an irreducible $G$-submodule of $C^{\infty}\left(L_{\lambda}\right)$ with the highest weight $\lambda$, hence if $\lambda \in Z_{c} \backslash\left(Z_{c}^{*} \cup\{0\}\right)$, then $\Gamma\left(L_{2}\right)=\{0\}$. It is known that $\lambda \in Z_{c}^{+}$if and only if $L_{\lambda}$ is very ample. Let $\mathscr{E}_{\nu}\left(L_{2}\right)$ be the eigenspace of $\Delta^{\lambda}$ with the eigenvalue $\nu$. The decomposition (2.2) of $C^{\infty}\left(L_{\lambda}\right)$ becomes

$$
C^{\infty}\left(L_{\lambda}\right)=\Gamma\left(L_{\lambda}\right)+\sum_{\nu>\nu(\lambda, \mu)} \mathscr{E}_{\nu}\left(L_{\lambda}\right) .
$$

Let $\phi=\varphi\left(V ; r_{1}, \cdots, r_{s}\right) ; M \rightarrow C P^{n}$ be a homogeneous map associated to a finite dimensional $G$-submodule $V$ of $C^{\infty}\left(L_{2}\right)$ as in Section 2. We use the same notation as in the preceding sections.

Proposition 4.2. (1) $\phi=\phi\left(V ; r_{1}, \cdots, r_{s}\right): M \rightarrow C P^{n}$ is a full holomorphic map if and only if $\lambda \in Z_{\mathrm{c}}^{:}$and $V=\Gamma\left(L_{\lambda}\right)(s=1)$.

(2) If $\lambda \in Z_{\mathrm{c}}$ satisfies $(\lambda, \alpha) \neq 0$ for each $\alpha \in \Pi-\Pi_{0}$ then $\varphi=$ $\varphi\left(V ; r_{1}, \cdots, r_{s}\right): M \rightarrow C P^{n}$ is an immersion.

(3) If $\lambda \in Z_{i}^{i}$ and $V \subset \mathscr{E}_{\nu}\left(L_{\lambda}\right)$ for $\nu>\nu(\lambda, \mu)$, then $\phi=\varphi\left(V ; r_{1}, \cdots, r_{s}\right)$ : $M \rightarrow C P^{n}$ is a harmonic map which is neither holomorphic, antiholomorphic nor totally real. Moreover if a harmonic map $\phi=\phi\left(V ; r_{1}, \cdots, r_{s}\right)$ : $M \rightarrow C P^{n}$ with the simple compact Lie group $G$ and $\operatorname{dim}_{c} M \geqq 2$ is neither holomorphic nor antiholomorphic, then $\phi$ is not even pluriharmonic (cf. [Ud], [O-U]).

(4) If $\varphi: M=G / K \rightarrow C P^{n}$ is a G-equivariant, stable harmonic map of a compact homogeneous Kähler manifold $M=G / K$ with the second Betti number $b_{2}(M)=1$, then $\phi$ is holomorphic or antiholomorphic.

Proof. (1) Note that by the $G$-invariance of $\phi$ and Lemma 3.1 we 
have $\left(D_{X} \Phi\right) \widetilde{\varphi}=X^{*} \widetilde{\Phi}$ for each $X \in T M^{c}$. Assume that $\lambda \in Z_{c}^{\sharp}$ and $V=\Gamma\left(L_{\lambda}\right)$ $(s=1)$. From $\lambda \in Z_{c}^{*}$ we see $\Gamma\left(L_{2}\right) \neq\{0\}$. Since $\left(\bar{\partial}_{\bar{Z}} \varphi_{i}\right)_{i=0, \cdots, n}=\left(\nabla_{\bar{Z}}^{\lambda} \varphi_{i}\right)_{i=0, \cdots, n}=$ $\bar{Z} * \widetilde{\Phi}=0$ for each $Z \in T^{(1,0)} M$, we get $D_{\bar{Z}} \Phi=0$. Thus $\phi$ is holomorphic. Conversely assume that $\phi$ is holomorphic. Then for each $Z \in T^{(1,0)} M$ we have $0=\left(D_{\bar{Z}} \Phi\right) \widetilde{\Phi}=\bar{Z}^{*} \widetilde{\Phi}=\left(\nabla_{\bar{Z}} \frac{\lambda}{\varphi_{i}}\right)_{i=0, \cdots, n}=\left(\bar{\partial}_{\bar{Z}} \varphi_{i}\right)_{i=0, \cdots, n}$. Hence $\left\{\varphi_{0}, \cdots, \varphi_{n}\right\}$ are nonzero holomorphic sections of $L_{\lambda}$. Thus by the Borel-Weil theorem we get $\lambda \in Z_{c}^{\#}$ and $V=\Gamma\left(L_{\lambda}\right)$.

(2) Let $\omega_{c}$ denote the Kähler form of $\left(C P^{n}, g_{c}\right)$. Then the first Chern class $c_{1}(H)$ of the hyperplane bundle $H$ over $C P^{n}$ is represented by $\gamma_{1}(H)=(c / 4 \pi) \omega_{c}$. Since $\phi^{*} c_{1}(H)=c_{1}\left(L_{\lambda}\right)$ and $\phi^{*} \gamma_{1}(H), \gamma_{1}\left(L_{\lambda}\right)$ are $G$ invariant closed 2-forms, by (4.2) and Proposition 4.1 we get $\varphi^{*} \gamma_{1}(H)=$ $\gamma_{1}\left(L_{2}\right)$, that is,

$$
\phi^{*} \omega_{o}=\frac{2}{c} \sqrt{-1} \sum_{\alpha \in \Sigma_{m}^{+}}(\lambda, \alpha) \omega^{\alpha} \wedge \bar{\omega}^{\alpha} .
$$

Hence we see that $(\lambda, \alpha) \neq 0$ for each $\alpha \in \Pi-\Pi_{0}$ if and only if $\varphi^{*} \omega_{c}$ is nondegenerate, and then $\varphi$ is an immersion.

(3) The first statement follows from (1). If $\varphi=\varphi\left(V ; r_{1}, \cdots, r_{s}\right)$ is a nonconstant pluriharmonic map, then by a result of [Ud] we have $\operatorname{rank}_{R} d \varphi \leqq 2$, hence $\operatorname{dim}_{R} \varphi(M)=1$ or 2. Since $G^{\prime}=\{a \in G ; a x=x$ for each $x \in \varphi(M)\}$ is a normal closed subgroup of $G$ and $G$ is simple, $G$ is locally isomorphic to $G / G^{\prime}$. Since $G / G^{\prime}$ acts effectively on $\varphi(M), G$ must be of type $\mathrm{A}_{1}$. Thus $\operatorname{dim}_{c} M=1$.

(4) By (2) $\varphi$ must be an immersion. By a result of [B-B] (see also [B-B-B-R]), we see that $\varphi(M)$ is a complex submanifold of $C P^{n}$. Hence by the $G$-equivariance of $\varphi$ the standard complex structure of $C P^{n}$ induces a $G$-invariant complex structure $J^{\prime}$ on $M$ through $\varphi$. By Proposition 13.8 of [B-H] the complex structure $J^{\prime}$ or $-J^{\prime}$ coincides with the complex structure $J$ of $M=G^{c} / U$. Thus $\varphi$ is holomorphic or antiholomorphic.

q.e.d.

REMARK. (1) The statement (1) of Proposition 4.2 can be regarded as a very special case of the famous Kodaira embedding theorem. If $\lambda \in Z_{\mathrm{c}}^{*} \backslash Z_{\mathrm{c}}^{+}$, then the holomorphic map $\varphi=\varphi\left(\Gamma\left(L_{\lambda}\right)\right): M \rightarrow C P^{n}$ is not an immersion. For each $\lambda \in Z_{\mathrm{c}}^{+}$, we have a Kähler embedding $\varphi=\varphi\left(\Gamma\left(L_{\lambda}\right)\right)$ : $(M,(4 \pi / c) g(\lambda)) \rightarrow\left(C P^{n}, g_{c}\right)$. These Kähler embeddings were investigated and classified in detail by [Te 2].

(2) If $(G, K)$ is an irreducible Hermitian symmetric pair, since the isotropy representation of $M=G / K$ is irreducible, the statement (3) of Proposition 4.2 gives neither holomorphic, antiholomorphic nor totally real, minimal isometric immersions $\varphi:\left(M, r g_{G}\right) \rightarrow C P^{n}$ for some $r>0$. The 
values $r$ and eigenspaces $\mathscr{E}_{\nu}\left(L_{\lambda}\right)$ are determined precisely from Proposition 3.4 and results of the next section.

§5. Spectral decomposition for complex line bundles over compact Hermitian symmetric spaces.

In this section we discuss the case when the generalized flag manifold $M$ is a Hermitian symmetric space.

Let $\Pi=\left\{\alpha_{1}, \cdots, \alpha_{l}\right\}$ be an irreducible Dynkin diagram. A pair $\left(\Pi, \Pi_{0}\right)$ is called irreducible symmetric if $\Pi-\Pi_{0}=\left\{\alpha_{i_{0}}\right\}$ and the highest root $\tilde{\alpha}$ of the system $\Sigma$ with the fundamental root system $\Pi$ has an expression:

$$
\tilde{\alpha}=\alpha_{i_{0}}+\sum_{i=1, i \neq i_{0}}^{l} m_{i} \alpha_{i}, \quad m_{i} \in Z, m_{i}>0 .
$$

A general pair $\left(\Pi, \Pi_{0}\right)$ of Dynkin diagrams is said to be symmetric if the pair $\left(\Pi, \Pi_{0}\right)$ is a direct sum of irreducible symmetric pairs of Dynkin diagrams. Let $M=G / K$ be a generalized flag manifold associated to an effective pair $\left(\Pi, \Pi_{0}\right)$. It is known that the pair $(G, K)$ is a symmetric pair if and only if the pair $\left(\Pi, \Pi_{0}\right)$ is symmetric. In this case for any $G$-invariant Riemannian metric $g$ on $M,(M, g)$ is a Hermitian symmetric space and the identity component $\operatorname{Aut}^{\circ}(M, g)$ of all automorphisms of $(M, g)$ is equal to $G$. Every Hermitian symmetric space of compact type is obtained in this way.

Proposition 5.1 (cf. [H-C], [He]). There exists a subset $\gamma_{1}, \cdots, \gamma_{r}$ of $\Sigma_{\mathrm{m}}^{+}$consisting of strongly orthogonal roots, i.e. $\gamma_{i} \pm \gamma_{j} \notin \Sigma(1 \leqq i, j \leqq r)$ such that

$$
\mathfrak{a}^{c}=\sum_{i=1}^{r} \boldsymbol{C}\left(E_{r_{i}}+E_{-r_{i}}\right)
$$

is a maximal abelian subspace of $\mathfrak{m}^{c}$. In particular

$$
\boldsymbol{a}=\sum_{i=1}^{r} \boldsymbol{R}\left(E_{r_{i}}+E_{-r_{i}}\right)
$$

is a maximal abelian subspace of $\mathfrak{m}$.

Consider the inner automorphism $\nu$ of the Lie algebra $g$, the so called Cayley transformation

$$
\nu=\operatorname{Ad}\left(c_{0}\right) \text {, }
$$

where $c_{0}=\exp (\pi / 2) \sqrt{-1} \sum_{i=1}^{r}\left(E_{\gamma_{i}}-E_{-\gamma_{i}}\right) /\left(\sqrt{2}\left|\gamma_{i}\right|\right)$. Then we have $\nu(a) \subset t$ and $\nu\left(\left(E_{\gamma_{i}}-E_{-r_{i}}\right) / \nu \overline{2}\right)=-\gamma_{i} /\left|\gamma_{i}\right|$ for $1 \leqq i \leqq r$ (cf. [H-C]). 
Set $\Lambda_{i}=\Lambda_{\alpha_{i}}$ for $i=1, \cdots, l$. In our case we have $Z_{\mathrm{c}}=\boldsymbol{Z} \Lambda_{i_{0}}$. For each $\lambda=k \Lambda_{i_{0}} \in Z_{\mathrm{c}}$, the first Chern class $c_{1}\left(L_{\lambda}\right)$ is given by $c_{1}\left(L_{\lambda}\right)=k \omega_{1}$ where $\omega_{1}$ is a positive generator of $H^{2}(M, Z)$. For each $k \in Z$, set $W_{k}=\left(\sigma\left(k \Lambda_{i_{0}}\right), \boldsymbol{C}\right)$.

We shall determine the spectral decompositions (2.1) of all complex line bundles over each irreducible Hermitian symmetric space of compact type. Let $D(G)$ be the set of all dominant integral elements of t. By Proposition 2.2 we know $\operatorname{dim} \operatorname{Hom}\left(V(\Lambda), W_{k}\right)=0$ or 1 for each $\Lambda \in D(G)$. Set

$$
D(G, K ; k)=\left\{\Lambda \in D(G) ; \operatorname{dim} \operatorname{Hom}_{K}\left(V(\Lambda), W_{k}\right)=1\right\}
$$

for each $k \in Z$, and $D(G, K)=D(G, K ; 0)$.

THEOREM 5.2. The following is a complete list of $D(G, K ; k)(k \in Z)$ for each compact irreducible Hermitian symmetric space $M=G / K$. Here each diagram is the Satake diagram of $(G, K)$ and ()) represents the element of $\Pi-\Pi_{0}$.

(1) (AIII) $)_{p q}(1 \leqq p \leqq q): M=G_{p, q}(C), l=p+q-1$. If $1 \leqq p \leqq l / 2$,

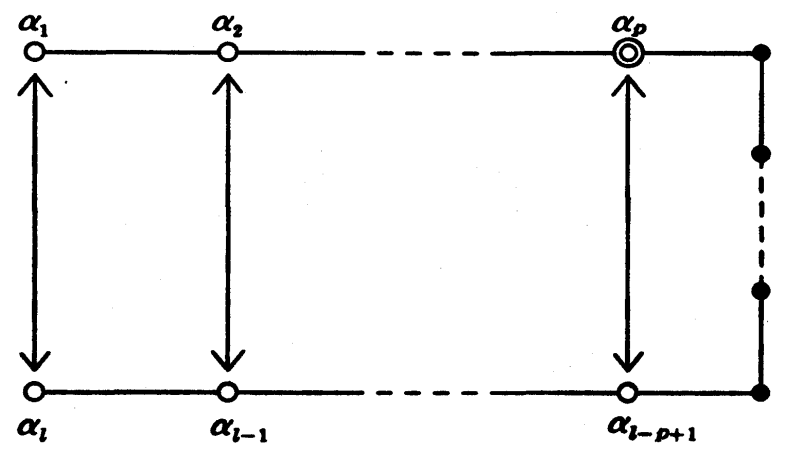

$$
\begin{aligned}
& D(G, K ; k)=\left\{\sum_{i=1}^{p-1} m_{i}\left(\Lambda_{i}+\Lambda_{l-i+1}\right)+m_{p} \Lambda_{p}+m_{p+1} \Lambda_{l-p+1} ;\right. \\
& \left.m_{i} \in Z, m_{i} \geqq 0(i=1, \cdots, p+1), m_{p}-m_{p+1}=k\right\} .
\end{aligned}
$$

If $2 p-1=l$,

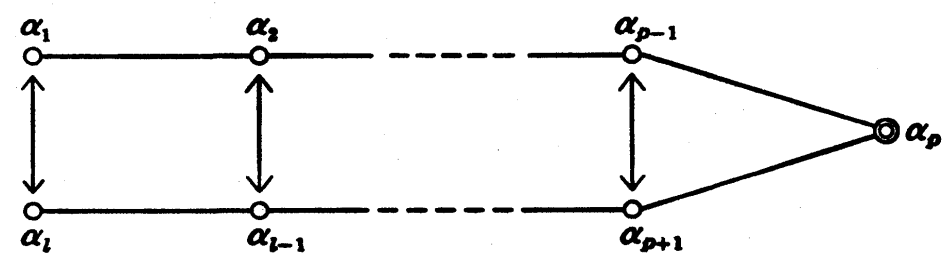

$$
\begin{aligned}
& D(G, K ; k)=\left\{\sum_{i=1}^{p-1} m_{i}\left(\Lambda_{i}+\Lambda_{l-i+1}\right)+m_{p} \Lambda_{p} ;\right. \\
& \left.m_{i} \in Z, m_{i} \geqq 0(i=1, \cdots, p), m_{p}-|k| \geqq 0 \text { is even }\right\} .
\end{aligned}
$$


(2) (BD) $)_{m}(m \geqq 5): M=Q_{m}(C), l=[m / 2]+1 \geqq 3$. If $m$ is odd $(l \geqq 3)$,

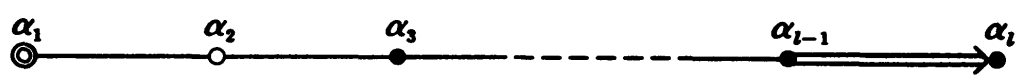

If $m$ is even $(l \geqq 4)$,

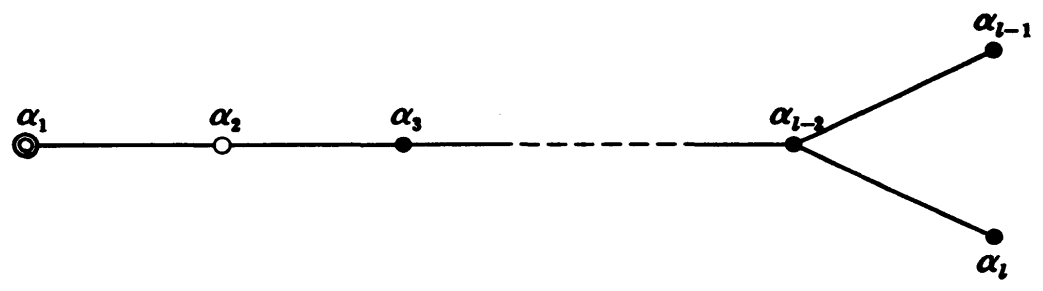

$D(G, K ; k)=\left\{m_{1} \Lambda_{1}+m_{2} \Lambda_{2} ;\right.$ $m_{i} \in Z, m_{i} \geqq 0(i=1,2), m_{1}-|k| \geqq 0$ is even $\}$.

(3) $(\mathrm{CI})_{l}(l \geqq 2): M=S p(l) / U(l)$.

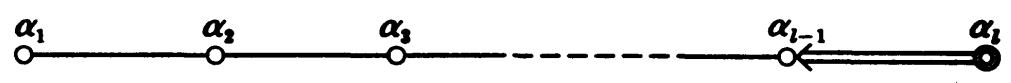

$D(G, K ; k)=\left\{\sum_{i=1}^{l-1} m_{i} 2 \Lambda_{i}+m_{l} \Lambda_{l} ;\right.$

$$
\left.m_{i} \in Z, m_{i} \geqq 0(i=1, \cdots, l), m_{l}-|k| \geqq 0 \text { is even }\right\} .
$$

(4) (DIII) ${ }_{l}(l \geqq 5) ; M=S O(2 l) / U(l)$. If $l=2 r$,

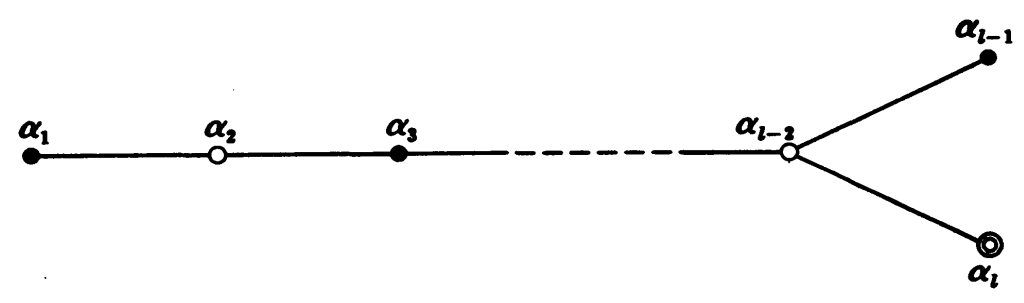

$$
\begin{aligned}
& D(G, K ; k)=\left\{\sum_{i=1}^{r-1} m_{i} 2 \Lambda_{2 i}+m_{r} \Lambda_{l} ;\right. \\
& \left.m_{i} \in Z, m_{i} \geqq 0(i=1, \cdots, r), m_{r}-|k| \geqq 0 \text { is even }\right\} .
\end{aligned}
$$

If $l=2 r+1$,

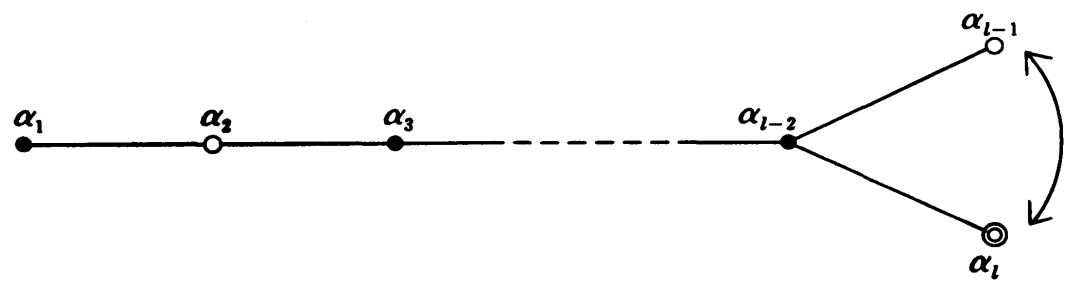




$$
\begin{aligned}
& D(G, K ; k)=\left\{\sum_{i=1}^{r-1} m_{i} 2 \Lambda_{2 i}+m_{r} \Lambda_{l-1}+m_{r+1} \Lambda_{l} ;\right. \\
& \left.m_{i} \in Z, m_{i} \geqq 0(i=1, \cdots, r+1), m_{r}-m_{r+1}=-k\right\} \text {. }
\end{aligned}
$$

(5) (EIII): $M=E_{8} / \operatorname{Spin}(10) \cdot T$.

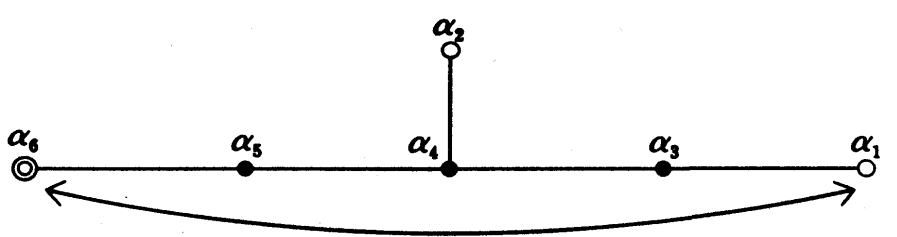

$$
\begin{aligned}
D(G, K ; k)= & \left\{m_{1} \Lambda_{1}+m_{2} \Lambda_{2}+m_{3} \Lambda_{8} ;\right. \\
& \left.m_{i} \in Z, m_{i} \geqq 0(i=1,2,3), m_{1}-m_{3}=-k\right\} .
\end{aligned}
$$

(6) (EVII): $M=E_{7} / E_{8} \cdot T$.

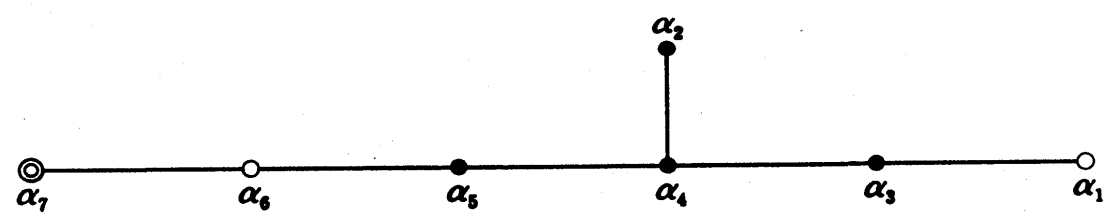

$$
\begin{aligned}
D(G, K ; k)= & \left\{m_{1} \Lambda_{1}+m_{2} \Lambda_{8}+m_{3} \Lambda_{7} ;\right. \\
& \left.m_{i} \in Z, m_{i} \geqq 0(i=1,2,3), m_{3}-|k| \geqq 0 \text { is even }\right\} .
\end{aligned}
$$

We will get Theorem 5.2 by proving the following lemma.

Lemma 5.3. Let $p$ be the Satake involution of $(G, K)\left(\right.$ or $\left.\left(\Pi, \Pi_{0}\right)\right)$.

(1) If $p\left(i_{0}\right) \neq i_{0}$, then

$$
\begin{aligned}
& D(G, K ; k)=D(G, K)+k \Lambda_{i_{0}} \quad \text { for each } k \geqq 0 \text { and } \\
& D(G, K ; k)=D(G, K)-k \Lambda_{p\left(i_{0}\right)} \quad \text { for each } k<0 .
\end{aligned}
$$

(2) If $p\left(i_{0}\right)=i_{0}$, then

$$
D(G, K ; k)=D(G, K)+|k| \Lambda_{i_{0}} \quad \text { for each } k \in \mathbb{Z} .
$$

Proof. Note that if $p\left(i_{0}\right) \neq i_{0}$, then $k \Lambda_{i_{0}} \in D(G, K ; k)$ for $k \geqq 0$ and $-k \Lambda_{p\left(i_{0}\right)} \in D(G, K ; k)$ for $k<0$, and if $p\left(i_{0}\right)=i_{0}$, then $|k| \Lambda_{i_{0}} \in D(G, K ; k)$ for $k \in Z$. Let $\Lambda \in D(G, K ; k)$. We show that if $k \geqq 0$, then $\Lambda \in D(G, K)-k \Lambda_{p\left(i_{0}\right)} \cdot$ Let $k$ be a nonnegative integer. Now we take the tensor product $G$ - 
module $\left(V\left(k \Lambda_{i_{0}}\right)\right)^{*} \otimes V(\Lambda)$. Here $V^{*}$ denotes the dual $G$-module of a $G$ module $V$. Then we have the direct sum decomposition of the $G$-module $\left(V\left(k \Lambda_{i_{0}}\right)\right)^{*} \otimes V(\Lambda)$ into irreducible $G$-modules $U_{i}$

$$
\left(V\left(k \Lambda_{i_{0}}\right)\right) * V(\Lambda)=U_{1} \oplus U_{2} \oplus \cdots \oplus U_{s},
$$

where $U_{1}$ denotes the highest component. Since $\left(V\left(k \Lambda_{i_{0}}\right)\right)^{*}=V\left(k \Lambda_{p\left(i_{0}\right)}\right)$, we have $U_{1}=V\left(k \Lambda_{p\left(i_{0}\right)}+\Lambda\right)$. Choose a nonzero weight vector $f \in\left(V\left(k \Lambda_{i_{0}}\right)\right)^{*}$ for the lowest weight $-k \Lambda_{t_{0}}$ and a nonzero vector $w \in V(\Lambda)$ belonging to a $K$ submodule of $V(\Lambda)$ isomorphic to $W_{k}$. Then we see $\rho(X) f=-\sqrt{-1}\left(k \Lambda_{t_{0}}, X\right) f$ for each $X \in \mathfrak{f}$ and $\rho(a)(f \otimes w)=f \otimes w$ for each $a \in K$, where $\rho$ denotes the action of $G$ on each representation space. Moreover choose a nonzero weight vector $f_{1} \in\left(V\left(k \Lambda_{i_{0}}\right)\right)^{*}$ for the highest weight $k \Lambda_{p\left(i_{0}\right)}$ and a nonzero weight vector $v_{1} \in V(\Lambda)$ for the highest weight $\Lambda$. Note that $f_{1} \otimes v_{1} \in U_{1}$. For each $H \in t$, we have

$$
\rho(H)\left(f_{1} \otimes v_{1}\right)=V \overline{-1}\left(k \Lambda_{p\left(i_{0}\right)}+\Lambda, H\right)\left(f_{1} \otimes v_{1}\right) .
$$

Since $\left\langle\rho\left(c_{0}\right)^{-1} f_{1}, f\right\rangle \neq 0$ and $\left\langle\rho\left(c_{0}\right)^{-1} v_{1}, w\right\rangle \neq 0$ by Lemma 2.1, we have

$$
\left\langle\rho\left(c_{0}\right)^{-1}\left(f_{1} \otimes v_{1}\right), f \otimes w\right\rangle=\left\langle\rho\left(c_{0}\right)^{-1} f_{1}, f\right\rangle\left\langle\rho\left(c_{0}\right)^{-1} v_{1}, w\right\rangle \neq 0 .
$$

Write $f \otimes w=u_{1}+\cdots+u_{s}$, where $u_{i} \in U_{i}(i=1, \cdots, s)$. Then we see $\rho(a) u_{i}=u_{i}$ for each $a \in K$ and $\rho\left(c_{0}\right)(f \otimes w)=\rho\left(c_{0}\right) u_{1}+\cdots+\rho\left(c_{0}\right) u_{s}, \rho\left(c_{0}\right) u_{i} \in U_{i}$ $(i=1, \cdots, s)$. Since $\left\langle f_{1} \otimes v_{1}, \rho\left(c_{0}\right)(f \otimes w)\right\rangle=\left\langle f_{1} \otimes v_{1}, \rho\left(c_{0}\right) u_{1}\right\rangle \neq 0$, we get $\rho\left(c_{0}\right) u_{1} \neq 0$. Hence $0 \neq u_{1} \in U_{1}$ and $\rho(a) u_{1}=u_{1}$ for each $a \in K$. Thus we obtain $k \Lambda_{p\left(i_{0}\right)}+\Lambda \in D(G, K)$.

Similarly we can show that if $k<0$ and $\Lambda \in D(G, K ; k)$, then $\Lambda \in$ $D(G, K)+k \Lambda_{i_{0}}$. We have only to apply the same argument to the tensor product $G$-module $V\left(-k \Lambda_{i_{0}}\right) \otimes V(\Lambda)$.

First we discuss the case when $p\left(i_{0}\right) \neq i_{0}$, that is, the pair $(G, K)$ is of type (AIII) $)_{p q}$, (DIII) $l_{l=2 r+1}$ or (EIII). Let $\Lambda \in D(G, K ; k)$. Assume $k \geqq 0$. By the above assertion we can write

$$
\Lambda=m_{0}^{\prime}\left(\Lambda_{i_{0}}+\Lambda_{p\left(i_{0}\right)}\right)-k \Lambda_{p\left(i_{0}\right)}+\left[\text { terms involving } \Lambda_{j} \text { with } j \neq i_{0}, p\left(i_{0}\right)\right]
$$

for some $m_{0}^{\prime} \in Z, m_{0}^{\prime} \geqq 0$. Hence we get

$$
\left.\Lambda=m_{0}\left(\Lambda_{i_{0}}+\Lambda_{p\left(i_{0}\right)}\right)+k \Lambda_{i_{0}}+\text { [terms involving } \Lambda_{j} \text { with } j \neq i_{0}, p\left(i_{0}\right)\right]
$$

with $m_{0}=m_{0}^{\prime}-k \geqq 0$. Thus we obtain $\Lambda \in D(G, K)+k \Lambda_{i_{0}}$. Next assume $k<0$. Similarly by the above assertion we can write

$$
\left.\Lambda=m_{0}^{\prime}\left(\Lambda_{i_{0}}+\Lambda_{p\left(i_{0}\right)}\right)+k \Lambda_{i_{0}}+\text { [terms involving } \Lambda_{j} \text { with } j \neq i_{0}, p\left(i_{0}\right)\right]
$$


for some $m_{0}^{\prime} \in Z, m_{0}^{\prime} \geqq 0$. Hence we get

$$
\Lambda=m_{0}\left(\Lambda_{i_{0}}+\Lambda_{p\left(i_{0}\right)}\right)-k \Lambda_{p\left(i_{0}\right)}+\left[\text { terms involving } \Lambda_{j} \text { with } j \neq i_{0}, p\left(i_{0}\right)\right]
$$

with $m_{0}=m_{0}^{\prime}+k \geqq 0$. Thus we obtain $\Lambda \in D(G, K)-k \Lambda_{p\left(i_{0}\right)}$. Conversely if $\Lambda^{\prime} \in D(G, K)$ and $k \geqq 0 \quad$ (resp. $\left.k<0\right)$, then $\Lambda^{\prime}+k \Lambda_{i_{0}}$ (resp. $\left.\Lambda^{\prime}-k \Lambda_{p\left(i_{0}\right)}\right) \in$ $D(G, K ; k)$. In fact, if we let $u \in V\left(\Lambda^{\prime}\right)$ and $v \in V\left(k \Lambda_{i_{0}}\right)$ (resp. $V\left(-k \Lambda_{p\left(i_{0}\right)}\right)$ ) a nonzero $K$-fixed element of $V\left(\Lambda^{\prime}\right)$ and the highest (resp. lowest) weight vector of $V\left(k \Lambda_{i_{0}}\right)$ (resp. $V\left(-k \Lambda_{p\left(i_{0}\right)}\right)$ ), then by Lemma 2.1 we see that the highest component of the tensor product $V\left(\Lambda^{\prime}\right) \otimes V\left(k \Lambda_{i_{0}}\right)$ (resp. $V\left(\Lambda^{\prime}\right) \otimes$ $\left.V\left(-k \Lambda_{p\left(i_{0}\right)}\right)\right)$ contains a nonzero component of $u \otimes v$, and hence it contains a $K$-submodule isomorphic to $W_{k}$. Therefore we obtain (1).

Next we proceed to the case when $p\left(i_{0}\right)=i_{0}$, that is, the pair $(G, K)$ is of type $(\mathrm{BD})_{m},(\mathrm{CI})_{l}$, (DIII) $)_{l=2 r}$ and (EVII). Let $\Lambda \in D(G, K ; k)$. From the above assertions we already know $\Lambda \in D(G, K)-|k| \Lambda_{i_{0}}$. Hence from Satake diagrams we can write

$$
\left.\Lambda=m_{0}^{\prime} 2 \Lambda_{i_{0}}-|k| \Lambda_{i_{0}}+\text { [terms involving } \Lambda_{j} \text { with } j \neq i_{0}\right]
$$

for some $m_{0}^{\prime} \in Z, m_{0}^{\prime} \geqq 0$. Thus it becomes

$$
\Lambda=m_{0} \Lambda_{i_{0}}+\text { [terms involving } \Lambda_{j} \text { with } j \neq i_{0} \text { ] }
$$

with $m_{0}=2 m_{0}^{\prime}-|k| \geqq 0$ and $m_{0}-|k|$ even. We have to show $m_{0} \geqq|k|$. By the assumption $V(\Lambda)$ contains a $K$-submodule isomorphic to $W_{|k|}$, which also denoted by $W_{|k|}$, because $V(\Lambda)^{*}$ is isomorphic to $V(\Lambda)$. By the irreducibility of $V(\Lambda)$ and the $K$-invariance of $W_{|k|}$, we have

$$
V(\Lambda)=\sum_{q=0}^{N}, \sum_{\beta_{1}, \cdots, \beta_{q} \in \Sigma_{\mathrm{m}}} \rho\left(\mathfrak{g}_{\beta_{1}}^{c}\right) \cdots \rho\left(\mathfrak{g}_{\beta_{q}}^{c}\right) W_{|k|}
$$

for a sufficiently large integer $N$. Note that $\rho\left(\mathfrak{g}_{\beta_{1}}^{c}\right) \cdots \rho\left(\mathfrak{g}_{\beta_{q}}^{c}\right) W_{|k|}$ is contained in the weight space of $V(\Lambda)$ for the weight $|k| \Lambda_{i_{0}}+\beta_{1}+\cdots+\beta_{q}$, or it is zero. Hence we can write

$$
\Lambda=|k| \Lambda_{i_{0}}+\beta_{1}+\cdots+\beta_{q}
$$

for some $q \geqq 0$ and $\beta_{1}, \cdots, \beta_{q} \in \Sigma_{\mathrm{m}}^{+}$. Indeed, we have only to choose $\left\{\beta_{1}, \cdots, \beta_{q}\right\} \subset \Sigma_{\mathrm{m}}$ of the minimal number $q$ such that $\rho\left(E_{\beta_{1}}\right) \cdots \rho\left(E_{\beta_{q}}\right) w$ is the nonzero highest weight vector of $V(\Lambda)$, where $w$ is a nonzero element of $W_{|k|}$. We check that

$$
\frac{2\left(\beta, \alpha_{i_{0}}\right)}{\left(\alpha_{i_{0}}, \alpha_{i_{0}}\right)}=0,1 \text { or } 2
$$

for each $\beta \in \Sigma_{\mathrm{m}}^{+}$. Indeed, we define an integer $j_{0}$ by $j_{0}=2$ if $(G, K)$ is of 
type $(\mathrm{BD})_{m}, j_{0}=l-1$ if $(G, K)$ is of type $(\mathrm{CI})_{l}, j_{0}=l-2$ if $(G, K)$ is of type (DIII) $)_{l=2 r}$ and $j_{0}=6$ if $(G, K)$ is of type (EVII). Then from the list of root systems we see that

$$
\left(\alpha_{i_{0}}, \alpha_{j}\right)=0 \text { for } j \neq i_{0}, j_{0}, \text { and } \frac{2\left(\alpha_{j_{0}}, \alpha_{i_{0}}\right)}{\left(\alpha_{i_{0}}, \alpha_{i_{0}}\right)}=-1,
$$

and for each $\beta \in \Sigma_{\mathrm{m}}^{+}, \beta$ has the following form:

$$
\beta=\left\{\begin{array}{l}
\alpha_{i_{0}} \\
\alpha_{i_{0}}+\alpha_{j_{0}}+\left[\text { terms involving } \alpha_{j} \text { with } j \neq i_{0}, j_{0}\right], \text { or } \\
\alpha_{i_{0}}+2 \alpha_{j_{0}}+\left[\text { terms involving } \alpha_{j} \text { with } j \neq i_{0}, j_{0}\right] .
\end{array}\right.
$$

Hence we get (5.2). Therefore from (5.1) we have $m_{0}=2\left(\Lambda, \alpha_{i_{0}}\right) /\left(\alpha_{i_{0}}, \alpha_{i_{0}}\right)=$ $|k|+2 \sum_{i=1}^{q}\left(\beta_{s}, \alpha_{i_{0}}\right) /\left(\alpha_{i_{0}}, \alpha_{i_{0}}\right) \geqq|k|$. We got $D(G, K ; k) \subset D(G, K)+|k| \Lambda_{i_{0}}$. In the same way as the proof of (1) we can show $D(G, K)+|k| \Lambda_{i_{0}} \subset D(G, K ; k)$. We obtain (2).

q.e.d.

Proof OF TheOREM 5.2. The method of determining $D(G, K)$ by Satake diagram is well-known in the theory of spherical functions over compact symmetric spaces (cf. [Te 1] and references of [He]). Therefore Theorem 5.2 follows from Lemma 5.3 .

q.e.d.

\section{§6. Classification of homogeneous harmonic maps between complex projective spaces.}

In this section we discuss the case when $M$ is an $n$-dimensional complex projective space $\boldsymbol{C P}^{m}$. By Theorem 5.2, Freudenthal's formula and Weyl's dimension formula, a simple computation gives the following.

Proposition 6.1. For the case (AIII) $)_{1 m}: M=C P^{m}$, for each $\Lambda=$ $m_{1} \Lambda_{1}+m_{2} \Lambda_{m} \in D(G, K ; k)$ with $m_{1}-m_{2}=k$, the eigenvalue $c(\Lambda)$ of the Casimir operator for $\Lambda$ relative to the inner product of $\mathfrak{g}=\mathfrak{B} \mathfrak{u}(m+1)$ defined by $(-1)$ times the Killing form, and the dimension $d(\Lambda)$ of the representation of $\mathfrak{b u}(m+1)$ with the highest weight $\Lambda$ are given as follows;

$$
\begin{aligned}
& c(\Lambda)=\frac{1}{2(m+1)^{2}}\left\{m m_{1}^{2}+2 m_{1} m_{2}+m m_{2}^{2}+m(m+1)\left(m_{1}+m_{2}\right)\right\}, \\
& c(\Lambda)-\left|k \Lambda_{1}\right|^{2}=\frac{1}{2(m+1)}\left\{2 m_{1} m_{2}+m\left(m_{1}+m_{2}\right)\right\}, \\
& d(\Lambda)=\left(\begin{array}{c}
m-1+m_{1} \\
m_{1}
\end{array}\right)\left(\begin{array}{c}
m-1+m_{2} \\
m_{2}
\end{array}\right) \frac{m+m_{1}+m_{2}}{m} .
\end{aligned}
$$


By this proposition and results of the previous sections we get a nice series of homogeneous harmonic maps between complex projective spaces.

THEOREM 6.2. There exists a series of $S U(m+1)$-equivariant full minimal isometric immersions $\psi_{n, l}^{m}: C P^{m}(c(n, l)) \rightarrow C P^{N(n, l)}(c)$ indexed by the set $\{(n, l) \in \boldsymbol{Z} \times \boldsymbol{Z} ; n \geqq l \geqq 0\}$, where

$$
\begin{aligned}
& c(n, l)=\frac{c m}{2 l(n-l)+m n}, \\
& N(n, l)+1=\left(\begin{array}{c}
n-l+m-1 \\
n-l
\end{array}\right)\left(\begin{array}{c}
l+m-1 \\
l
\end{array}\right) \frac{m+n}{m} .
\end{aligned}
$$

Moreover $\left\{\psi_{n, l}^{m}\right\}$ satisfy the following:

(1) $l=0$ if and only if $\psi_{n, l}^{m}$ is holomorphic. In this case $\psi_{n, l}^{m}$ is the $n$-th Veronese imbedding of $\mathrm{CP}^{m}$ (cf. [Te 2]).

(2) $l=n$ if and only if $\psi_{n, l}^{m}$ is antiholomorphic.

(3) $n$ is even and $2 l=n$ if and only if $\psi_{n, l}^{m}$ is totally real. In this case $\psi_{n, l}^{m}$ is a composite of the l-th standard minimal immersion $\boldsymbol{C P}^{m} \rightarrow$ $\boldsymbol{S}^{N}(c / 4)$ (cf. [Wa 1]), the natural isometric covering $S^{N}(c / 4) \rightarrow R P^{N}(c / 4)$ and the totally real totally geodesic imbedding $\boldsymbol{R} P^{N}(c / 4) \rightarrow C P^{N}(c)$.

(4) The degree of $\psi_{n, l}^{m}$ is equal to $n-2 l$.

REMARK. In case $m=1, \psi_{n, l}^{1}$ is congruent to $\psi_{n, l}$ in Theorem 1 of [B-O] (cf. [B-J-R-W], [Gu 1]). So this theorem is just a generalization of the result of $[\mathrm{B}-\mathrm{O}]$ to higher dimensional complex projective spaces.

Proof. For each $\Lambda=m_{1} \Lambda_{1}+m_{2} \Lambda_{m} \in D(G, K ; k)$, we set $\psi_{n, l}^{m}=\varphi\left(V_{A}\right)$ $(s=1)$ by Proposition 3.3, where $n=m_{1}+m_{2}, l=m_{2}$ and $k=n-2 l$. Then we get the above series.

Theorem 6.3. Let $\varphi: C P^{m} \rightarrow C P^{N}$ be a full $S U(m+1)$-equivariant harmonic map between complex projective spaces with the Fubini-Study metrics. Then there exists a pair of integers $(n, l)$ with $0 \leqq l \leqq n$ such that $N=N(n, l)$ and $\phi$ is equivalent to $\psi_{n, l}^{m}$.

REMARK. From this result we see immediately that if $\varphi: C P^{m}\left(c^{\prime}\right) \rightarrow$ $C P^{N}(c)$ is a full $S U(m+1)$-equivariant minimal isometric immersion, then there exists a pair $(n, l)$ with $0 \leqq l \leqq n$ such that $c^{\prime}=c(n, l), N=N(n, l)$ and $\varphi$ is congruent to $\psi_{n, l}^{m}$.

Proof. Let $k$ be the degree of $\varphi$. By the results of Section 3, there exists a unique homogeneous complex line bundle $L$ with $c_{1}(L)=k$ 
over $C P^{m}$ such that $\varphi$ is equivalent to $\varphi\left(V ; r_{1}, \cdots, r_{s}\right)$ for some $G$-submodule $V$ of an eigenspace of $\Delta^{L}$ in $C^{\infty}(L)$ and some $r_{1}, \cdots, r_{s} \in R$ with $\sum_{i=1}^{i} r_{i}^{2}=1$. It suffices to show $s=1$. Let $V=\sum_{i=1}^{s} V^{(i)}$ be the direct sum decomposition into irreducible $G$-modules and $\Lambda^{(i)}=m_{1}^{(i)} \Lambda_{1}+m_{2}^{(i)} \Lambda_{m} \in D(G, K ; k)$ be the highest weight of $V^{(i)}$ for $i=1, \cdots, s$. Then we have

$$
\begin{cases}m_{1}^{(i)}-m_{2}^{(i)}=k & \text { for } \quad i=1, \cdots, s, \\ c\left(\Lambda^{(i)}\right)=c\left(\Lambda^{(i+1)}\right) & \text { for } \quad i=1, \cdots, s-1 .\end{cases}
$$

By (6.1) and Proposition 6.1, a simple computation shows $m_{1}^{(i)}=m_{1}^{(i+1)}$ and $m_{2}^{(i)}=m_{2}^{(i+1)}$ for $i=1, \cdots, s-1$, that is, $\Lambda^{(i)}=\Lambda^{(i+1)}$ for $i=1, \cdots, s-1$. By Corollary 2.3, we get $s=1$. q.e.d.

REMARK. (1) It is important to investigate the rigidity of the above minimal immersions. Refer to [Ca] for the rigidity of $\psi_{n, 0}^{m}$ or $\psi_{n, n}^{m}$ and to [Wa 1], [Ur] for the rigidity of $\psi_{n, l}^{m}$ with $n=2 l$.

(2) After the author finished this work, Dr. Burstall informed him that Toth also got examples of non(anti)holomorphic harmonic maps $C P^{m} \rightarrow C P^{n}$ for $m<n$.

\section{§7. Homogeneous minimal 2-spheres in quaternionic projective spaces.}

It is interesting to study homogeneous harmonic maps into quaternionic projective spaces by applying our argument to quaternionic line bundles and smooth maps into quaternionic projective spaces. Here we give homogeneous minimal 2-spheres in quaternionic projective spaces and some information about them. They were discussed first by Salamon [Sa].

Let $\boldsymbol{H} P^{n}(c)$ denote an $n$-dimensional quaternionic projective space with the standard metric of maximum sectional curvature $c$.

Proposition 7.1. There exists a series of SU(2)-equivariant minimal isometric immersions $\varphi_{n, \alpha}: \boldsymbol{C P}(c(n, \alpha)) \rightarrow \boldsymbol{H} P^{n}(c)$ indexed by the set $\{(n, \alpha) \in Z \times Z ; n \geqq \alpha \geqq 0\}$, where

$$
\begin{aligned}
& c(n, \alpha)=\frac{c}{2 \alpha(2 n+1-\alpha)+2 n+1} \quad \text { for } \alpha=1, \cdots, n-1, \\
& c(n, n)=\frac{c}{n(n+1)},
\end{aligned}
$$

and the image of each $\varphi_{n, \alpha}$ is not contained in any proper totally geodesic submanifold of $\boldsymbol{H} P^{n}$. Moreover $\alpha=0$ or $n$ if and only if $\varphi_{n, \alpha}$ is totally 
complex. Conversely they give all proper SU(2)-equivariant minimal immersions of $\boldsymbol{C} P^{1}$ into $\boldsymbol{H} P^{n}$.

REMARK. (1) Refer to [Ts] for the definition of a totally complex immersion. It is equivalent to the inclusive condition in [E-S] as a smooth map. In [Sa] a connection of these maps with the twistor spaces $S p(n+1) / S p(n) \times U(1)$ and $S p(n+1) / U(n) \times S p(1)$ over $\boldsymbol{H} P^{n}$ was studied. This result shows that the statements in Lemma 2.10 and Theorem 4.1 in [Ts] do not hold for $\varphi_{n, n}$.

(2) We conjecture that $\left\{\varphi_{n, \alpha}\right\}$ exhaust all proper minimal isometric immersions of $\boldsymbol{C} \boldsymbol{P}^{1}\left(c^{\prime}\right)$ into $\boldsymbol{H} P^{n}(c)$.

ACKNowledgement. The author would like to thank Professor K. Ogiue for constant encouragement, Dr. S. Maeda (Kumamoto Institute of Technology, Japan) for suggestion of a problem on minimal immersions between complex projective spaces, and Dr. M. A. Guest (Rochester, USA), Dr. S. Udagawa for stimulating conversation and interest in this work. The author was supported by the Max-Planck-Institut für Mathematik in Bonn during the preparation of this paper. He would like to thank the referee for his careful reading of the manuscript.

\section{References}

[B-O] S. BANDo and Y. OHNITA, Minimal 2-spheres with constant curvature in $P_{n}(C)$, J. Math. Soc. Japan, 39 (1987), 477-487.

[B-J-R-W] J. Bolton, G. R. Jensen, M. Rigoli and L. M, Woodward, On conformal minimal immersions of $S^{2}$ into $C P^{n}$, Math. Ann., 279 (1988), 599-620.

[B-H] A. BOREL and F. HIRZEBRUCH, Characteristic classes and homogeneous spaces I, Amer. J. Math., 80 (1958), 458-538.

[Bt] R. Вотт, Homogeneous vector bundles, Ann. of Math., 58 (1957), 203-248.

[B-B] D. BURNS and P. DE BARTOLOMEIS, Applications harmoniques stables dans $P^{n}$, Ann. Sci. Ecole Norm. Sup., 21 (1988), 159-177.

[B-B-B-R] D. BURNS, F. BURSTAll, P. DE Bartolomeis and J. RAwnsley, Stability of harmonic maps of Kähler manifolds, J. Diff. Geom., 30 (1989), 579-594.

[Ca] E. CALABI, Isometric imbeddings of complex manifolds, Ann. of Math., 58 (1953), 1-23.

[E-L] J. Eells and L. Lemaire, Another report on harmonic maps, Bull. London Math. Soc., 20 (1988), 385-524.

[E-S] J. EELLS and S. SALAMON, Constructions twistorielles des applications harmoniques, C.R. Acad. Sci. Paris, 296 (1983), 685-687.

[E-W] J. EELLS and J.C. WooD, Harmonic maps from surfaces to complex projective spaces, Adv. in Math., 49 (1983), 217-263.

[Gu 1] M. A. GUEST, Geometry of maps between generalized flag manifolds, J. Diff. Geom., 25 (1987), 223-247.

[Gu 2] M. A. GUEST, Instantons and harmonic maps, Infinite Dimensional Groups with 
Applications, edited by V. Kac, Math. Sci. Res. Inst. Publ., Vol. 4 (1985), SpringerVerlag, 137-156.

[Gu 3] M. A. GUEST, Orbits and harmonic maps, Contemporary Math., 58, Part III (1987), 161-171.

[Gu 4] M. A. GUEST, The energy of harmonic maps obtained by the twistor construction, Max-Planck-Institut für Math., preprint 86-50.

[Gu 5] M. A. GUEST, The energy function and homogeneous harmonic maps, preprint.

[H-C] HARISH-ChANDRA, Representations of semi-simple Lie groups, VI, Amer. J. Math., 78 (1956), 564-628.

[He] S. Helgason, Differential Geometry, Lie Groups and Symmetric Spaces, Academic Press (1978).

[Hu] S. HUMPHREYs, Introduction to Lie Algebras and Representation Theory, Revised, Springer-Verlag (1972).

[K-N] S. KobaYASHI and K. Nomizu, Foundations of Differential Geometry I, II, WileyInterscience $(1963,1969)$.

[Oh 1] Y. OHNITA, On stability of minimal submanifolds in compact symmetric spaces, Composition Math., 64 (1987), 157-189.

[Oh 2] Y. OHNITA, On pluriharmonicity of stable harmonic maps, J. London Math. Soc., 35 (1987), 563-568.

[O-U] Y. OHNITA and S. Udagawa, Stability complex-analyticity and constancy of pluriharmonic maps from compact Kähler manifolds, to appear in Math. Z.

[Sa] S. Salamon, Harmonic and holomorphic maps, Geometry Seminar "Luigi Bianchi" II-1984, Lecture Notes in Math., 1164 (1985), 161-224, Springer-Verlag.

[Ta] T. TAKAhashi, Minimal immersions of Riemannian manifolds, J. Math. Soc. Japan, 18 (1966), 380-385.

[Te 1] M. TAKEUCHI, Modern Theory of Spherical Functions, Iwanami, Tokyo (1975), in Japanese.

[Te 2] M. TAKEUCHI, Homogeneous Kähler submanifolds in complex projective spaces, Japan. J. Math., 4 (1978), 171-219.

[Ts] K. Tsurada, Parallel submanifolds in a quaternionic projective space, Osaka J. Math., 22 (1985), 187-241.

[Ud] S. UDAGAWA, Pluriharmonic maps and minimal immersions of Kähler manifolds, J. London Math. Soc., 37 (1988), 375-384.

[Ur] H. URAKAwa, Minimal immersions of projective spaces into spheres, Tsukuba J. Math., 9 (1985), 321-347.

[Wa 1] N. R. WALlach, Minimal immersions of symmetric spaces into spheres, Symmetric Spaces, (W. B. Boothby and G. L. Weiss, eds.), Marcel Dekker (1972), 1-40.

[Wa 2] N. R. W ALLACH, Harmonic Analysis on Homogeneous Spaces, Marcel Dekker (1973).

[Wo] J.A. WoLF, The geometry and structure of isotropy irreducible homogeneous spaces, Acta Math., 120 (1968), 59-148. Correction; Acta Math., 152 (1984), 141-142.

Present Address:

Department of Mathematics, Tokyo Metropolitan University

Fukasawa, Setagaya-kU, Tokyo 158, Japan 